\title{
Delay Tolerant Network assisted flying Ad-Hoc network scenario: Modeling and analytical perspective
}

\author{
Amartya Mukherjee \\ Dept. of Computer Science \&Engineering \& BSH, \\ Institute of Engineering and Management, Salt Lake, Kolkata, India \\ amartya.mukherjee@iemcal.com \\ Nilanjan Dey \\ Department of Information Technology, \\ Techno India College of Technology, Kolkata, India \\ nilanjan.dey@tict.edu.in \\ Rajesh Kumar \\ Faculty of Electrical Engineering, \\ M.N.I.T, Jaipur, India \\ rkumar.ee@mnit.ac.in \\ B. K Panigrahi \\ Dept.of Electrical Engineering, \\ Indian Institute of Technology, Delhi, India \\ bkpanigrahi@ee.iitd.ac.in \\ Aboul Ella Hassanien \\ Faculty of Computers and Information, Cairo University, Cairo, Egypt, \\ \& Faculty of Computer and Information, Beni Suef University, Beni Suef, \\ Egypt \& Scientific Research Group in Egypt (SRGE), Cairo, Egypt \\ aboitcairo@gmail.com \\ João Manuel R.S. Tavares \\ Instituto de Ciência e Inovação em Engenharia Mecânica e Engenharia Industrial, Departamento de \\ Engenharia Mecânica, Faculdade de Engenharia, Universidade do Porto, Porto, Portugal \\ tavares@fe.up.pt
}

\begin{abstract}
Flying Ad-Hoc networks (FANET) are the extended paradigm of the mobile Ad-Hoc networks and, perhaps, one of the most emerging research domains in the current era. A huge number of tangible applications have been developed in this domain. The main advantages of such networks are their easy deployment, scalability, and robustness. However, the sparseness of these networks is an inherent characteristic that is known to be a bottleneck. The main objective of this work was to provide an alternative solution for the intermittently connected FANET by considering the philosophy of the Delay Tolerant Network (DTN) approach. To realize the functionality of the DTN protocols in a threedimensional (3D) space, a social FANET model is proposed. FANET nodes are supposed to have a sparse node density. Fundamentally, the proposed DTN assisted Flying Ad-hoc Network exploits the DTN routing and mobility features. The new mobility modeling for 3D spaces was re-engineered and tested with well-known routing protocols to analyze the performance of the model based on node speed, density,
\end{abstract}


buffer, latency, message overhead, and power consumption. The effectiveness of 3D mobility models has also been compared against the one of classical models. The obtained results reflect a significant enhanced performance of the suggested DTN protocol for sparse FANET in a social scenario.

Keywords: FANET, DTN, Routing, Mobility Model, Delivery Probability, Latency, Energy.

\section{Introduction}

Flying Ad-Hoc networks (FANET) are collaborative and organized collections of Unmanned Aircraft Systems (UAS). FANET can be widely used to establish wireless network infrastructures in areas like disaster-hit regions, control borders or lines of territories where there are no pre-established network connections or suffers a complete shutdown due to an unexpected catastrophe. The node of a FANET is a dynamic flying machine, mainly a fixed-wing drone or a Multicopter having Vertical Takeoff and Landing (VTOL) capability. In the case of Multicopter, Quad and Hexcopter models are highly preferred due to their high stability. The node does not have a human pilot on board, hence is controlled by some autopilot unit, and powered through batteries of high charge density. Such drones can be built very easily by means of open hardware design [1,2], like the Ardupilot Mega, Multiwii, and Pixhawk drones. These nodes can be manually or automatically maneuverable. In the case of the fully autonomous nodes, the formation of swarms is very common depending upon the requirement of the topology and the pattern of the mobility [3, 4]. Architecturally, a Flying Ad-Hoc network is a layered approach where the node is at the bottom layer and is either a solitary or a cluster of multiple nodes. There are multiple possible node formations; perhaps the most popular amongst them is the cluster of the node that includes a cluster head which sometimes works as a master node. The UAVs in a swarm performs multi-hop communication, where each node acts as hop count or a relay that forwards the data packet towards the next node available in the queue. The forwarding of messages or data packets is predefined through several routing mechanisms. At the physical layer, where the node performs its mobility and also do the data transmission in the form of Wi-Fi (IEEE802.11 b/g/n) or Zigbee medium, the fundamental control of the data transmission and the routing of the data packet is, therefore, the job of the Medium Access Control (MAC) layer, which acts just on the top of the physical layer. The MAC layer of FANET provides a methodology where the layer is open for all nodes in the existing group of nodes. Therefore, there is a high chance to occur the collision of data packets. To avoid such a situation, network models have been proposed [5]. One of the most popular mechanisms is the contention based MAC protocol, where the neighboring node competes to share through the random access. In the case of contention free technique, the medium is not governed by the random access but synchronized by some scheduling mechanism. The protocol like directional antenna based MAC provides the leverage in FANET structure to enhance the end to end connectivity and bypass the difficulties of link failure. A novel approach of position prediction based MAC protocol [6] can be addressed in this context, where the position of each FANET node is predicted based on a GPS vector. Several network layer routing protocols have also been developed for efficient routing of data packets. A topology broadcast based on reverse path forwarding protocol [7] is another approach for the intra-cluster communication between two or more nodes. Intelligent UAVAssisted Routing Protocol for Urban VANETs [8, 9, and 10] is also a well-known research approach, where searches for the shortest path through a UAV assisted routing protocol can be performed. For a densely deployed FANET architecture, the greedy forwarding protocol $[11,12]$ is also applicable, which 
optimizes the number of hops during the transition of the message from a source to destination. In this case, the fundamental principle is to choose the geographically closest node for forwarding the message. UAV Ad-Hoc network (UAANET) is another immerging concept [13], which is a subset of the standard mobile ad-hoc network where is mainly emphasized the direct communication from UAV to Ground Control Station (GCS) and from UAV to UAV as well. The primary disadvantage of such methodology is the huge amount of bandwidth that should be dedicated for each UAV to communicate since as the number of node increases, the communication requirements also increase. Cellular network based communication has also been proposed in this context; however, it is highly expensive and never gives a tangible solution. An Intelligent MAC (IMAC) [14], or hybrid MAC protocol, has also been developed and works both in the network and MAC layer in order to ensure a better end to end delivery in an IEEE802.11 interface. Beaconless Opportunistic Routing [15] is a geographical beaconless protocol that imbibes multimedia dissemination with a Quality of Experience support. Strong emphasizes on the MAC, Network layer protocol and their effects on the node mobility [16] have been given in a precise and specific manner. Dynamic Source routing [17, 18] is another routing protocol, which is a classical protocol and can be actively used in flying ad-hoc networks. In this case, the only drawback is the massive packet latency due to the discovery of the multiple routes at a time. A Comparison of Stateless Position-based Packet Routing Algorithms for FANETs [19] has also been proposed, where the node can be identified by its location, rather than based on its address, and the data packets get forwarded based on their coordinates.

This study was developed to overcome the following well-known problems and disadvantages. A FANET model with high node density has some major impact on several elements of the message transfer. If the node density is high, the message overhead will increase. In most of the cases, buffer overflow is obvious during the flooding of the messages. If the number of nodes increases, the cumulative energy consumption of the model will also increase. It is also observed that for Ad-hoc networks the increasing node density leads to degradation of data packets delivery and throughput and increases end-to-end delay [20]. Therefore, in the specific applications, the use of FANET model with a high node density is not preferable, especially in challenging tasks like wireless infrastructure networks in borders and lines of control zones; building a network infrastructure over a vast ocean or a desert; the exploration and establishment of a network infrastructure in an extraterrestrial zone, like the Mars surface, where the payload of the space probe is a big issue and sparseness of node is a normal phenomenon. Therefore, the main contribution of this work involves the redesign of the mobility model of the Delay Tolerant Networks (DTN) that performs the mobility of the node in a three-dimensional (3D) space to support FANET. The developed solution gives the direction for the thought of an alternative approach such that the node of the FANET can exploit the inherent behavior of a DTN node.

The remainder of the article is organized in the following way: Section 2 presents a detail literature review of the relevant and contemporary related work. Section 3presentsthe state-of-the-art concept related to the DTN assisted FANET architecture and details the proposed extended mobility design in the context of social FANET. Section 4describesthe experimental setup and presents the results analysis, which was carried out based on performance metrics; the performance of the redefined mobility models is discussed. Section 5 involves the energy analysis of the proposed routing methodology and mobility model. Finally, the conclusion is drawn in Section 6. 


\section{Related works}

There are several developed works considering the mobility of FANET as the crucial issue to be focused on. The architecture of the network is deployed in such a way that the nodes are dense enough and follow some specific movement pattern within a specified geographical region. The simplest mobility pattern that can be observed in a group of UAVs is the Zig-zag pattern, which is followed to perform, for example, a raster scan image acquisition [21]. The most classical and perhaps fundamental mobility models are the Random waypoint and Random walk models [22, 23, and 24]. The Random waypoint model is a very common Entity-Based mobility model where each mobile node randomly selects one point as its destination and travels towards this destination with constant velocity chosen uniformly and randomly in $\left[0, V_{\max }\right]$. Upon reaching the destination, the node stops for a duration defined by the 'pause time' parameter $\left(T_{\text {pause }}\right)$. After this duration, it again chooses another random destination and moves towards it. A random walk can be considered as a special case of Random Waypoint model where the pause time is zero. In this model, the nodes change their direction and speed after a certain time interval. Each and every node randomly and uniformly chooses its new direction $\theta(t)$ in $[0,2 \pi]$ for every new interval $t$. Similarly, a new speed, $v(t)$, is chosen uniformly in $\left[0, V_{\max }\right]$. Thus, during any time interval $t$, a node moves with the velocity vector $(v(t) \cdot \cos \theta(t), v(t) \cdot \sin \theta(t))$. If a node moves and touches the boundary of the area under study, it gets bounced back to the interior of the area with an angle of $\theta(t)$ or $\pi$ $-\theta(t)$. The main challenge in such case is to enhance the mobility of those models in a 3D space precisely.

A specific mobility model for disaster management has been proposed by Mukherjee et al. [25] where two types of node are considered. The first category of $x$ nodes among $n$ nodes performs a random movement by following Random waypoint and Random walk within a cluster that covers the disaster-hit region, and the remainder $n-x$ number of nodes will be at the cluster boundary and are responsible for establishing the connectivity with GCS. Path planned mobility like Paparazzi mobility model [26] is also considered as a stochastic model built upon the concept of the state machine and supports waypoint, oval, scan and eight formations, where an aerial vehicle can choose a randomized speed. The Gauss-Markov (GM) [27] model is another time space-based mobility model that takes the previous direction and the speed to compute the new values in each iteration. The extended 3DGauss-Markov model [28], which includes the additional $z$-axis, is also applicable to FANET mobility. Here, the third variable keeps track of the pitch of the node with respect to the horizon. In those cases, the mobility of the nodes is predictable and nodes are trying to make a permanent link between the network and GCS. Practical Swarm Mobility has also been proposed by Li et al. [29] where the PSO algorithm is implemented in order to optimize the problem by iteratively trying to improve the given candidate solution with respect to a given measured quality. In this case, the movement of each node is highly influenced by its local positions as well as it is guided into a better-known position within the search space and update that better location founded by another neighboring node. They also proposed a group mobility model called Spatio-Temporally correlated Group Mobility, fundamentally based on the Gauss Markov model [30]. The model emphasizes the temporal property of trajectory as well as spatial correlation about the multiple UAVs that are able to fly in a coordinated manner. In another work, Bouachir et al. [31] have made a comparative study between Paparazzi mobility and Random waypoint mobility and have found a tradeoff between end to end delivery and the delivery ratio. In another work, Radu et al. [32] give the direction to bridge up the FANET application to a Fog System that could increase the potential chance of the rescue of mobility form in a disaster-hit region. MP-OSLR routing strategy with standard mobility models has also been 
suggested. Rosati et al.[33] have proposed a new routing strategy known as P-OSLR and compare it to the standard OSLR strategy. They considered a GPS guided map based high-speed movement model and analyze the performance of the routing only. Michael Le (2006) [34] addressed a UAV assisted Disruption Tolerant Network focused mainly on the design and evaluation of routing mechanism for the highly partitioned network. The considered scenario is a vast geographically separated region (two islands) where there is no connection between them. The work addressed a real-life GPS information trajectory calculation during route discovery and data forwarding. In another work, Mohamed-Ayoub Messous [35] proposed a novel mobility approach that covers energy constraint, UAV network connectivity as well as area coverage. The developed concept mainly emphasizes the mobility modeling of the autonomous UAV fleet. The fellowship weight parameter $\alpha$ is the key to the proposed approach which decides whether one UAV will follow its adjacent UAV node or not. The model is said to be a new approach and achieve global convergence and fairness in comparison to two well-known models. The concept of Last mile Tactical Edge Network (TEN)[36] tries to overcome the limitation of the resource constraint equipment carried by the soldiers and has been highlighted in the form of software related delay tolerant approach. The integrated communication device has the most suitable means of communication and comprises mini UAVs, piezo-electric sensors and small communication devices that not depends upon the infrastructure network like 4G/5G.Sánchez-García et al. [37] proposed a PSO based navigation for UAVs in a disaster like a scenario. The algorithm is able to perform faster trajectory formation to identify victims, but it fundamentally considers an ideal mobility scenario where nodes are frequently connected to victims, which is not a realistic approach. Karmakar et al.[38]introduced a modified BFS technique to design a message transfer protocol for AUV systems. Due to the high space complexity (of about $O\left(n^{l}\right)$ ), this method will definitely suffer from high buffer usage, which is not desired for FANET nodes.

The motivation of this work encompasses alternative mobility and routing solution for Flying Ad-Hoc Network for the intermittently connected and challenged network, which sacrifices the performance in term of message delivery, latency, node density, overhead ratio, and power consumption. To the best of our knowledge, no such attempt of modification of FANET models has been proposed according to a delay tolerant network perspective, which can be seen as the main novelty of this work. In addition to that, this work is an effort to create a tangible solution that provides a wireless infrastructure for uninterrupted Internet service, real-time traffic update, interplanetary and deep space network infrastructure, to be used in weather forecast for those regions that are geographically separated with a significant amount of distance and don't have a good infrastructure to deploy Vehicular Ad-hoc Networks.

\section{DTN assisted FANET concept}

The fundamental background of the FANET research is to achieve good message transmission speed, reduce the transmission latency, optimize power consumption and improve the node mobility patterns. The algorithms that have been proposed so far present good performances where the deployment location is not enough vast and most of the nodes are in the line of sight with each other. The density of the nodes in such case is also comparatively high. The communication from one node to another can easily be performed in such a high node density; however, the real problem is the sparseness of the network. Since there is a tradeoff between nodes density and the cumulative energy dissipation of entire FANET [39], in a social scenario, like a big city where the different points have been separated by vast geographical 
distance, the standard FANET routing protocols are therefore compromised. One solution is to increase the number of nodes, but, by doing so; the performance of the network in terms of message overhead and cumulative energy will be drastically reduced [40]. Under these circumstances, Delay Tolerant Network, which is sometimes known as Disruption Tolerant Network (DTN), is the only solution through which the data packet can be delivered with good performance, i.e. good Delivery Ratio, even when the network is intermittently connected.

This work, therefore, addresses a DTN assisted solution for Flying Ad-Hoc networks where the mobility pattern and the routing of DTN get mapped in Flying Ad-hoc network scenarios. The standard routing algorithms use DTN assisted routing FANET where the mobility models take the vital role. The mobility models, in this case, were redesigned to serve the purpose of the intermittently connected UAV nodes on the fly.

\subsection{Social FANET scenario}

Social FANET is a group of UAV nodes that serves in a geographical region. The name social has been used because there is an expectation that the nodes of the FANET will perform a synchronized and collaborative task that mimics the social behavior of the system. The nodes have to ensure that they will never interrupt the task of the other nodes and they perform the cooperative goal. In this architecture, the nodes are intermittently connected with each other and perform opportunistic flooding or forwarding [41, 42] of the data packets. There are numerous flooding and forwarding methodologies reported in this context. For example, the direct contact method where each node comes into direct contact with each other to perform message exchanges. This method is supposed to be the degenerated cast of the forwarding and uses only one copy of the messages at a time. Another example is the tree-based flooding, which is similar to the diffusion computation technique and performs two-hop relay.

The most relevant protocol in the context of the social network scenario is the Exchange based routings where message exchange plays a crucial role. In this approach, the most effective routing algorithm for DTN was taken into account. The used routing strategy can be based on:

- Epidemic Routing: This is a classical routing strategy, perhaps the most simplified technique that can be applied to a DTN environment [43]. The working principle of the routing scheme is:

1. Initialize the message and queued it in a buffer;

2. Assign an ID to the message;

3. If contact happens:

a. Exchange the list of ID as a summary vector;

b. Check summary vector for undelivered message ID;

4. Transfer undelivered messages.

This methodology takes the advantage of the physical locality and supports both non-random and random movements of the nodes.

- Prophet Routing: This is a probabilistic routing protocol that uses history and transitivity phenomena [44]. The fundamental working principle of the Prophet routing involves a metric named delivery predictability $P(X, Y)$, according to $0 \leq P(X, Y) \leq 1$, and computed among every node $X$ and each known node $Y$. This metric is then computed in such a way that a node having a higher value for a specific destination will be chosen to be a better candidate to deliver the packet to the destination. On the other hand, if $P(X, Y)>P(Z, Y)$, then there is a better chance to send the 
data packet from $X$ to $Y$ rather than $Z$ to $Y$. Therefore, this protocol considers the following:

1. Each time nodes interact, the nodes exchange the summary vector and update their own entry based on the identity of the other node and the following equation is used for receiving:

$$
P(X, Y)=P(X, Y)_{o l d}+\left(1-P(X, Y)_{o l d}\right) \times P_{e n c}
$$

where $P_{\text {enc }}$ is a configurable encounter parameter.

2. If $X$ meets more frequently with $i$, then there is a good chance to route the message through $X$ to $i$, then from $i$ to $Y$, the destination. This is done based on:

$$
P(X, i)=P(X, i)_{\text {old }}+\left(1-P(X, i)_{\text {old }}\right) \times P(X, i) \times P(X, Y)
$$

3. To manage symmetry, the DP value gets aged for all $i$ with $\gamma$, where $\gamma$ is a constant aging factor, $T$ is the time, and the used procedure is:

$$
P(X, i)=P(X, i)_{\text {old }} \times \gamma^{T}
$$

- Spray and wait Routing: In this case, the message is transmitted with two phases for $L$ nodes [45]:

1. In the spray phase, a number of $L$ messages is forwarded, perhaps the nodes receiving a copy to $L$ distinct relay.

2. If it is unable to find the destination in the spray phase, each of the $L$ nodes carrying a single copy of the message performs the direct transmission.

- MaxPropRouting: The MaxProp Routing strategy is a novel approach to increase the chance of delivering messages to the destination [49]. There are three fundamental tasks that have to be carried out by MaxProp. 1) Neighbor discovery, 2) Data transfer and 3) storage management. The core of MaxProp follows a peer list of messages based on the cost assigned. Here, the cost is considered to be the delivery likelihood, and the adopted mechanism is given as:

1. Consider $n$ as the number of nodes, each node $i \in n$ keeps track of the meeting probability of its peer $j \in n$.

2. Set $f_{j}^{i} \leftarrow \frac{1}{|n|-1}$, where $f_{j}^{i}$ is the connection likelihood

3. If $i$ encounters $j$, then

$$
f_{j}^{i} \leftarrow f_{j}^{i}+1
$$

4. Compute the cost $c(i, i+1, \ldots d)$ to each path possible along destination $d$ :

$$
c(i, i+1, \ldots d)=\sum_{x=i}^{d-1}\left(1-\left(f_{x+1}^{x}\right)\right)
$$

5. Compute the cost of destination:

$$
c(d) \leftarrow \operatorname{low}[c(i, i+1, \ldots d)]
$$

Besides a routing scheme, FANET also needs a proper mobility model selection to support social structure. Since most of the existing DTN mobility modeling approaches upkeep movement in the two dimensional (2D) space ( $x$ and $y$-axis), it is quite challenging to realize the actual FANET scenario in this case. Thus, an enhanced mobility model is proposed to address the 3D space. Three typical movement models have been considered here, namely: Random Waypoint, Random Walk and Shortest Path Map based mobility with extended 3D navigation features. This work, therefore, is one of the newest attempts to attain the DTN model in Flying Ad-hoc network ecosystem.

\subsection{DTN assisted mobility design for FANET}


Here, as already aforementioned, three fundamental mobility models were considered and applied for the Flying Ad Hoc networks: Random Walk, Random waypoint, and Shortest Path Map based mobility models, which are classical models used in this area [46, 47]. Additionally, it was exploited the fundamental phenomena of those models to synthesize the modified model for 3D space. The mathematical models of these three-movement models are presented in the following.

The random waypoint modeling in 3D space can consider $\mathrm{a} \times b \times c$ cubic region and several random variables can be defined in order to represent the creation coordinate of the waypoints where $j$ node chooses the movement period of $i$, which can be denoted by vector $P_{i}{ }^{j}$. With this convention, the movement of the random waypoint model can be described as a stochastic process having discrete time. Therefore, for each time period $i$, we have:

$$
\left\{P_{i}^{j}\right\}_{i \in N_{0}}=P_{0}^{j}, P_{1}^{j}, P_{2}^{j}, P_{3}^{j} \cdots \cdots
$$

If it is assumed that the node will choose the random velocity of $V_{i}$ to reach from $P_{i-1}$ to $P_{i}$ with a pause time $T p$, then it can be written as:

$$
\left\{P_{i} V_{i} T_{p i}\right\}_{i \in N_{0}}=\left(P_{1} V_{1} T p_{1}\right)\left(P_{2} V_{2} T p_{2}\right)\left(P_{3} V_{3} T p_{3}\right) \ldots
$$

The value of the pause time is chosen from a random distribution $f T_{p}\left(\tau_{p}\right)$ having an interval of [0, $\left.\tau_{\text {pmax }}\right]$ with $\tau_{\text {pmax }}<\infty$. The velocity $V i$ is also chosen from a random distribution $F_{V}(v)$ within the interval $\left[\mathrm{V}_{\min }, \mathrm{V}_{\max }\right]$.

The transition length of the stochastic process is therefore addressed by:

$$
\left\{L_{i}^{j}\right\}_{i \in N_{0}}=P_{0}^{j}, P_{1}^{j}, P_{2}^{j}, P_{3}^{j} \ldots . .(10)
$$

with, $\quad L_{i}^{j}=\left\|P_{i}^{j}-P_{(i-1)}^{j}\right\|$

The Random Walk model is fundamentally influenced by the Gauss-Markov modeling. This model is a simple but effective mobility modeling that can be easily extended from $2 \mathrm{D}$ to $3 \mathrm{D}$ space. The limitations in a harsh environment, like the limited transmission range of the airborne networks, can be easily addressed with this model. The main feature of the model is that it is a memory based model having a tuning parameter $\alpha$. In this case, the Markov process is applied in a 3D space with $x_{n}, y_{n}, z_{n}$ axis for $n$ number of nodes with $3 \mathrm{D}$ velocity vector computed as:

$$
\begin{aligned}
& x_{n}=\alpha \cdot x_{n-1}+(1-\alpha) x^{\prime}+\sqrt{\left(1-\alpha^{2}\right)} x_{x_{n-1}} \\
& y_{n}=\alpha \cdot y_{n-1}+(1-\alpha) y^{\prime}+\sqrt{\left(1-\alpha^{2}\right)} y_{y_{n-1}} \\
& z_{n}=\alpha \cdot z_{n-1}+(1-\alpha) z^{\prime}+\sqrt{\left(1-\alpha^{2}\right)} z_{z_{n-1}}
\end{aligned}
$$

where $\alpha$ is the tuning parameter. The condition $\alpha=0$ implies a nonpredictable movement; hence the node is movement is memoryless. On the other hand, when it is equal to 1 (one), the movement is predictable. The direction $d_{n}$, distance factor $s_{n}$ and pitch $p_{n}$ can be obtained therefore, using the following equations, respectively:

$$
d_{n}=\alpha \cdot d_{n-1}+(1-\alpha) d^{\prime}+\sqrt{\left(1-\alpha^{2}\right)} d_{x_{n-1}}
$$




$$
\begin{aligned}
& p_{n}=\alpha \cdot p_{n-1}+(1-\alpha) p^{\prime}+\sqrt{\left(1-\alpha^{2}\right)} p_{x_{n-1}} \\
& s_{n}=\alpha \cdot s_{n-1}+(1-\alpha) s^{\prime}+\sqrt{\left(1-\alpha^{2}\right)} s_{x_{n-1}}
\end{aligned}
$$

Finally, the velocity vector along the three axes can be obtained by the following expressions:

$$
\begin{aligned}
& V_{x}=s_{n} \cos \left(d_{n}\right) \cos \left(p_{n}\right) \\
& V_{y}=s_{n} \sin \left(\mathrm{d}_{\mathrm{n}}\right) \cos \left(p_{n}\right) \\
& V_{z}=s_{n} \sin \left(p_{n}\right)
\end{aligned}
$$

In the case of shortest path map based mobility, the fundamental idea was derived based on the Dijkstra's shortest path finding algorithm. Hence, the movement path of each node is considered as a weighted graph $G=(V, N, w)$ with a cost function $w: E \rightarrow N$ that maps each edge $(u, v) \in E$ to a non negative integer, which implies a weight $w(u, v) \in N$. The length function can be extended in $w: V \times V \rightarrow N \cup\{\infty\}$ by considering $w(u, u)=0$ for all values of $u \in v$, considering $w(u, v)=\infty$ for all $(u, v)$ not belonging to $E$; therefore, it is considered as a directed path $x_{0} e_{1} x_{1} e_{2} \ldots . x_{n-l} e_{n}$, where $u=x_{0} \in V$ to $v=x_{n} \in V$ for graph $G=(V, N, w)$.Then, the length of path $\{L\}$ is:

$$
\{L\}=\sum_{i=1}^{n} w\left(x_{i-1}, x_{i}\right)
$$

As the movement of each node is decided with the graph mapping, the domain of vertices involved in the movement is small. The area of movement is defined using a specified map that mimics the property of the graph. The nodes movements are defined according to the vertices point. Hence, the Dijkstra shortest path algorithm can be used to predict the shortest path that each node should take to move from one point to another. As the complexity of Dijkstra's Algorithm is $O(e \log n)$, i.e. logarithmic time where $e$ is the number of edges and $n$ is the number of nodes, the number of vertices involved in the movement procedure is small and limited. Hence, this will yield a better a result as compared to other shortest path algorithms that are available.

By exploiting the above mathematical model, the recreated mobility models can be outlined. The modified Random walk model follows the Gauss-Markov rule as in Algorithm 1.

Algorithm 1: Random walk

1. Input:initial_range_x,initial_range_y,initial_range_z, and $\mathrm{d}_{\mathrm{n}}, \mathrm{p}_{\mathrm{n}}$.

2. current_x $\leftarrow$ current position in $\mathrm{x}$ axis;

3. current_y $\leftarrow$ current position in y axis;

4. current_z $\leftarrow$ current position in $\mathrm{z}$ axis;

5. min_distance $\leftarrow$ the minimum distance from one node to another;

6. max_distance $\leftarrow$ the maximum distance from one node to another;

7.n: number of nodes, $\alpha$ : tuning parameter, df $>0$ to get sparseness.

8. Output: x_new, y_new, z_new;

9. begin

10. step1:[Initialize the $x, y, z$ position parameters] 
11. $\mathrm{x}_{\mathrm{n}} \leftarrow \alpha \times \mathrm{x}_{\mathrm{n}-1}+(1-\alpha) \times \mathrm{x}^{\prime}+\sqrt{ }\left(1-\alpha^{2}\right) \mathrm{x}_{\mathrm{n}-1} \quad\{\alpha \leftarrow 1$; for better predictability $\}$

12. $\mathrm{y}_{\mathrm{n}} \leftarrow \alpha \times \mathrm{y}_{\mathrm{n}-1}+(1-\alpha) \times \mathrm{y}^{\prime}+\sqrt{ }\left(1-\alpha^{2}\right) \mathrm{y}_{\mathrm{n}-1}$

13. $\mathrm{z}_{\mathrm{n}} \leftarrow \alpha \times \mathrm{z}_{\mathrm{n}-1}+(1-\alpha) \times \mathrm{z}^{\prime}+\sqrt{ }\left(1-\alpha^{2}\right) \mathrm{z}_{\mathrm{n}-1}$

14. $\quad \mathrm{x} \leftarrow$ initial_range_ $\mathrm{x} \times$ current_ $\mathrm{x} \times \mathrm{x}_{\mathrm{n}}$

15. $\mathrm{y} \leftarrow$ initial_range_y $\times$ current_y $\times \mathrm{y}_{\mathrm{n}}$

16. $\mathrm{z}$ <initial_range_z $\times$ current_z $\times \mathrm{z}_{\mathrm{n}}$

17. step 2: [Computing the distance]

18. initial_range_d $=\alpha \cdot \mathrm{d}_{\mathrm{n}-1}+(1-\alpha) \mathrm{d}^{\prime}+\sqrt{ }\left(1-\alpha^{2}\right) \times \mathrm{d}_{\mathrm{n}-1}$

19. initial_range_p $=\alpha . \mathrm{p}_{\mathrm{n}-1}+(1-\alpha) \mathrm{p}^{\prime}+\sqrt{ }\left(1-\alpha^{2}\right) \times \mathrm{p}_{\mathrm{n}-1}$

$20 \mathrm{df} \leftarrow \alpha \cdot \mathrm{df}_{\mathrm{n}-1}+(1-\alpha) \mathrm{df}+\sqrt{ }\left(1-\alpha^{2}\right) \times \mathrm{df}_{\mathrm{n}-1} \quad$; compute distance factor

21. angle_d $\leftarrow 2 \times \pi \times$ initail_range_d ; to get direction angle

22. angle_p $\leftarrow 2 \times \pi \times$ initail_range_p ; to get pitch angle

23. distance $\leftarrow$ min_distance + initail_range $\times\left(\max \_\right.$distance - min_distance $)+\mathrm{df}$

24. step 3: [Compute the new position]

25. $\quad \mathrm{x} \_$new $\leftarrow \mathrm{x}+$ distance $\times \cos ($ angle_d $) \times \cos ($ angle_p $)$

26. $\quad$ y_new $\leftarrow \mathrm{y}+$ distance $\times \sin ($ angle_d $) \times \cos ($ angle_p $)$

27. $\quad$ z_new $\leftarrow z+$ distance $\times \sin ($ angle_p $)$

28. end

On the other hand, the random waypoint model takes a random initial co-ordinate in 3D space and starts the movement from that location. Then, each and every time as it reaches to its target coordinate; it simply takes another random coordinate in 3D space. Initially, the algorithm starts from the last updated waypoint position, which is supposed to be the initial arming position of the node. The factor initial range acts as a range factor for the navigation from the initial position to the final position, which must be greater than zero. The high value of initial range results in a random coordinate that may scatter within a large geographical distance. The new random coordinate position is computed by multiplying initial range with the last updated coordinate value, as depicted by Algorithm 2.

Algorithm 2: Random waypoint

1. Input: coordinate $\mathrm{x}, \mathrm{y}, \mathrm{z}$, initial_ranage;

2. Initial_coordinte_x $\leftarrow$ random $(x)$; 
3. Initial_coordinate_y $\leftarrow$ random $(y)$;

4. Initial_coordinate_z $\leftarrow$ random $(z)$;

5. Output: new_coordinate_x,new_coordinate_y,new_coordinate_z.

6. begin

7. step1: [set initial position]

8. last_waypoint_x $\leftarrow$ Initial_coordinte_x

9. last_waypoint_y $\leftarrow$ Initial_coordinte_y

10. last_waypoint_z $\leftarrow$ Initial_coordinte_z

\section{1. step 2:}

12. set initial_range $>0$

13. step 3: [Compute the new position]

14. new_coordinate_x $\leftarrow$ initial_range $\times$ last_waypoint_x

15. new_coordinate_y $\leftarrow$ initial_range $x$ last_waypoint_y

16. new_coordinate_z $\leftarrow$ initial_range $\times$ last_waypoint_z

\section{7. end}

The third movement model is the shortest path map based movement and fundamentally works according to the Dijkstra's shortest path algorithm. It finds the shortest path amongst all reachable paths from the source node and finds the most feasible path. In this case, we again have to initialize the coordinate with the initial arming position of the correspondent FANET node. After that, for all nodes $n$, the unvisited next coordinate is set to NULL. Therefore, all previous coordinates are set to NULL. Now, the previous coordinate set and unvisited coordinate set are inserted into the queue. To find out the new coordinate, the minimum weighted path has to be computed. In order to do that, the front element of the queue is removed and stored into $X 1$. Then, it should be compared with the alternate path, and if it is found to be longer than the alternate path, then this path is ignored, otherwise is considered. The implementation is in 3D space depicted by Algorithm 3.

Algorithm 3: Shortest path map based movement

1. Input: coordinates $\mathrm{x}, \mathrm{y}, \mathrm{z} ; \mathrm{n}$ : number of nodes;

2. Output: new_path, new_coordinate_x, new_coordinate_y,new_coordinate_z;

\section{3. begin}

4. step1: [Initialize the coordinates] 
5. initial_coordinte_x $\leftarrow x$;

6. initial_coordinate_y $\leftarrow y$;

7. initial_coordinate_z $\leftarrow \mathrm{z}$;

8.step 2: for all $\mathrm{n}$

10. Unvisited (initial_coordinte_x $\leftarrow$ NULL

11. Unvisited (initial_coordinte_y) $\leftarrow$ NULL

12. Unvisited (initial_coordinte_z) $\leftarrow$ NULL

13. V1 $\leftarrow$ [Unvisited (Initial_coordinte_x), Unvisited (initial_coordinte_y),

14. Unvisited (Initial_coordinte_z)]

15. $\mathrm{X} \leftarrow$ Previous_coordinate $([$ initial_coordinate_x, initial_coordinate_y,-

initial_coordinate_z ])

16. $\mathrm{V} 2 \leftarrow \mathrm{X}$

17. $\quad$ Queue $\leftarrow(\mathrm{V} 1, \mathrm{~V} 2)$

18. $\quad$ Distance $(\mathrm{V} 1, \mathrm{~V} 2) \leftarrow 0$

19.step 3: [Compute the new path]

20. while not empty Queue

21. value $($ new_coordinate_x, new_coordinate_y,new_coordinate_z $) \leftarrow$ remove $($ Queue $)$

22. $\mathrm{X} 1 \leftarrow$ value $($ new_coordinate_x, new_coordinate_y,new_coordinate_z)

23. For each Neighbor (X1)

24.Alt_path $(\mathrm{x}, \mathrm{y}, \mathrm{z}) \leftarrow$ Distance $(\mathrm{V} 1, \mathrm{~V} 2)+\operatorname{cost}(\mathrm{V} 1, \mathrm{~V} 2)$

$25 . \quad$ If Alt_path $(\mathrm{x}, \mathrm{y}, \mathrm{z})<\mathrm{X} 1$

26. new_path $\leftarrow$ Alt_path $(\mathrm{x}, \mathrm{y}, \mathrm{z})$

27. else

28. new_path $\leftarrow \mathrm{X} 1$

29. previous(new_path) $\leftarrow \mathrm{V} 1$

30. $\quad$ Distance $(\mathrm{V} 1, \mathrm{~V} 2) \leftarrow$ new_path

31. end while

\section{2. end}

\section{Simulation Setup and performance analysis}

The complete experiment was performed using the Opportunistic Network Environment (ONE) simulator version 1.5.1 [48]. This simulator is an agent-based simulation engine that supports discrete events, and whose main objective is to model the inter-node communication, node mobility, and message management. The ONE simulator provides a realistic testbed for evaluating a real-life application of DTN and mimics the convergence layer like Bluetooth, TCP, and UDP. The real-time integration from simulation to emulation support can be easily performed and emulate most of the aspect of the DTN such as radio link character, buffer storage constraints, mobility, and routing. Here, the idea of the FANET modeling was to execute the performance analysis of DTN assisted Flying Ad-Hoc networks. The 
simulation parameters were imputed as a script file in the format of 'option = value'; these parameters were chosen in such a way that the simulation mimics the most realistic scenario of a normal working day in an urban or a rural region separated by a considerable geographical distance, where a node can serve as an uninterrupted service provider of Internet, traffic updates and weather forecast.

Two crucial parameters that need to be addressed were node speed and node density. Hence, the impact of these parameters on the message delivery probability was required to be carefully studied. In scenarios like FANET, for example, where the nodes are far from each other and separated by a large amount of geographical distance, these two parameters are the key factors to ensure the chances of successful message delivery. In DTN, we mostly consider that the network is sparse. Therefore, an upper and lower benchmark of the nodes number must be taken into account in the network for optimal performance of the routing strategy. Some studies revealed that the increasing node density impacts in a reverse way on message delivery [51]. However, the node speed acts in a proportional way: It seems that a higher node speed results in a diminished message delivery due to a low encounter time amongst nodes, but the number of contacts between nodes per unit time is raised; nevertheless, the delivery ratio is increased [52]. In sections 4.2.1 and 4.2.2this issue is further discussed.

The energy sources for the nodes were supposed to be separate power units which are used for message transmission and scanning of the nodes. The energy consumption by each node due to scan and transmission was solely related to the routing algorithms, which have a uniform random distribution in this case. It was also considered that each node has a constant amount of energy source for driving the motor and other equipment on board. Therefore, the energy model that is used by the routing model involves a scan energy reduction $E_{s}$ and a transmission energy reduction $E_{t}$. Actually, the transmission energy $E_{t}$ can be obtained as:

$$
E_{t}=\Delta \times E_{x}
$$

Where $E_{x}$ is the energy consumption for transmission and $\Delta$ is a parameter whose value can be obtained by subtracting current updated time $T_{u}$ with the initial time of the node $T_{n}$ :

$$
\Delta=T_{n}-T_{u}
$$

Finally, the scan energy reduction can be obtained by the following formula where $E_{x s}$ is the required scan energy:

$$
E_{s}=\left\{\begin{aligned}
E_{x s} \times \Delta & \text { if } \Delta<0 \\
E_{x s} & \text { otherwise }
\end{aligned}\right.
$$

So the total energy reduced during routing is $E_{\text {sum }}=E_{s}+E_{t}$

Table 1. Used standard simulation parameters

\begin{tabular}{|l|l|}
\hline Parameter & Value \\
\hline
\end{tabular}




\begin{tabular}{|c|c|}
\hline Simulation Time & 43200 (12 hours) \\
\hline Number of FANET nodes & 40 \\
\hline $\begin{array}{l}\text { Transmission range for } \\
\text { IEEE } 802.11 \mathrm{~b} / \mathrm{g} / \mathrm{n} \text { interface }\end{array}$ & $100 \mathrm{~m}$ \\
\hline Transmission Speed & $250 \mathrm{~K}$ to $10 \mathrm{M}$ \\
\hline Standard Buffer Size & $500 \mathrm{M}$ \\
\hline Update Interval & $1 \mathrm{sec}$ \\
\hline Message TTL & 300 minute \\
\hline Simulation scenario Area & Metropolitan city scenario (180 Sq Km) \\
\hline Variation of Node Speed & $1 \mathrm{kmph}$ (i.e. walking speed) $-20 \mathrm{kmph}$ \\
\hline Variation of Message buffer size & $\begin{array}{l}\text { 6MB (node with low processing and } \\
\text { memory power) }-700 \mathrm{MB} \text { (high } \\
\text { processing node) }\end{array}$ \\
\hline$\alpha-$ value(for random walk) & 1 \\
\hline
\end{tabular}

Table 2. Used specific simulation parameters for routing protocol

\begin{tabular}{|c|c|}
\hline Parameters & Value \\
\hline Prophet (seconds in time unit) & $30 \mathrm{sec}$ \\
\hline Spray and wait (number of copies) & 3 \\
\hline Spray and wait (binary mode) & True \\
\hline Epidemic & N/A \\
\hline MaxProp & N/A \\
\hline
\end{tabular}

Table 1 indicates the used standard simulation parameters of the mobility models. On the other hand, Table 2 presents the simulation parameters for different routing models, i.e. the specific simulation parameters. Figure 1 depicts a snapshot for nodes movement in the proposed scenario using the flying Ad-Hoc network infrastructure for a specific instance of time. Each point is basically a FANET node that follows a specific mobility model. Each node is identified by a unique ID. The green circles indicate the transmission range of each node. When two nodes are in the transmission range of each other, the 
messages get transmitted between hem. For each node, a dotted stack representation is shown in order to mimic the operation of the message buffer.

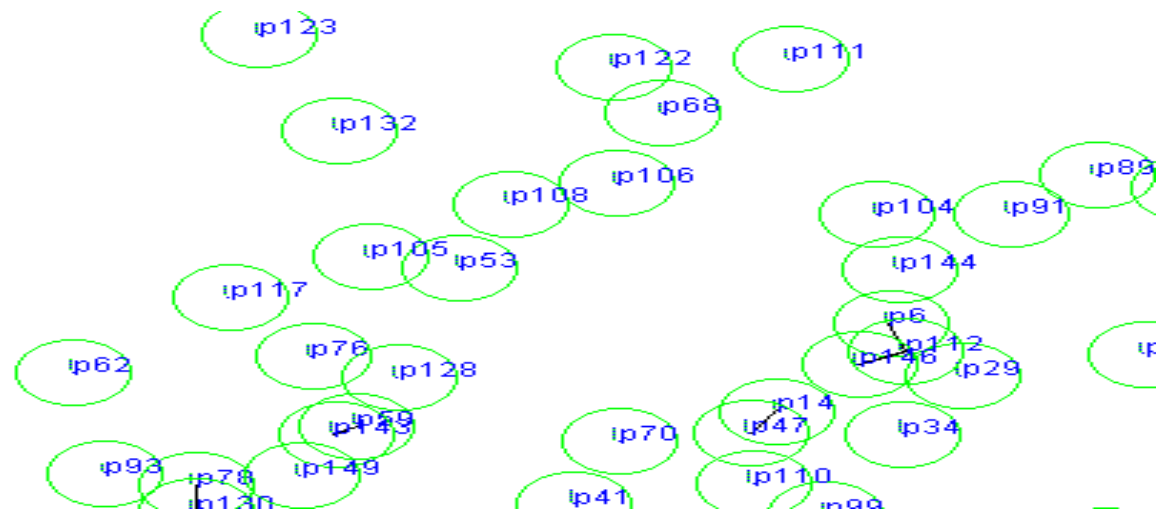

Fig 1. Snapshot of the used simulation environment.

\subsection{Performance metrics}

There are two crucial parameters that were chosen to be analyzed. First one is about the fly speed of the FANET nodes, node density and the buffer size of the FANET nodes. We elected these parameters because it is found that the major performance overhead of the FANET network suffers due to the varying non-synchronize speed of the nodes, and this is even more crucial in an intermittently connected FANET. The message delivery chance is highly affected due to the varying speed. It has been also reported that, depending upon the movement model, the performance also changes. FANET nodes are often suffered for a restricted buffer size, because of much less load carrying potentiality; therefore, the buffer size has to be taken as a crucial feature.

The performance metrics that were chosen to analyze the accomplishment of the routing methodology with a proliferated node mobility structure were: delivery probability, average latency, overhead ration and average buffer time.

The delivery probability $P(d)$ is expressed as the number of messages delivered upon the number of messages created:

$$
P(d)=\frac{M_{d}}{M_{c}}
$$

Where $M_{d}$ is the number of messages delivered and $M_{c}$ the number of messages created.

The Overhead $\left(O_{R}\right)$ ratio can be computed by the difference between relayed and delivered messages upon the delivered messages:

$$
O_{R}=\frac{\left(M_{r}-M_{d}\right)}{M_{d}}
$$

Where $M_{r}$ is the number of messages relayed and $M_{d}$ the number of messages delivered. 
Average Latency $(L)$ is the difference between the average time to deliver the $i^{\text {th }}$ message and the message buffered into the message buffer:

$$
L=T(i)_{d}-T(i)_{b}
$$

Finally, the Buffer time average $\left(B_{T}\right)$ signifies the difference between the time taken by $i^{\text {th }}$ message to exit and enter into the buffer memory:

$$
B_{T}=T(i)_{e x}-T(i)_{e n}
$$

\subsection{Result analysis about the delivery probability under proposed mobility models}

\subsubsection{Performance of node speed vs. delivery probability}

Figure 2 depicts the impact of the delivery probability for each movement model corresponding to each specific routing protocol under study.

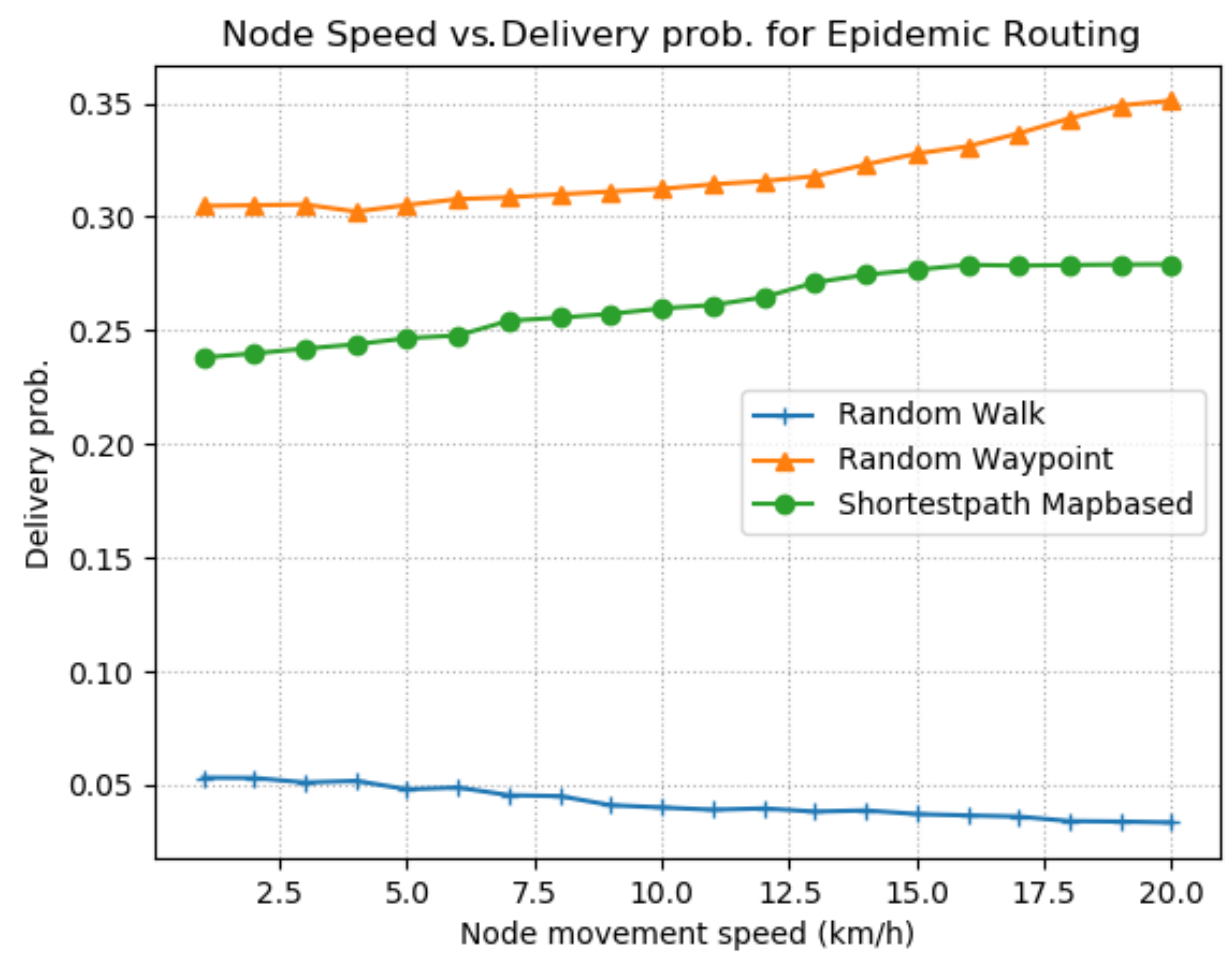

Fig 2. Node speed vs. Delivery Probability: epidemic routing for the proposed Random Walk, Random Waypoint, and Shortest path Map based movement models.

Figure 2 illustrates the effect of the node speed on the delivery of the packets to the destination. From the graph shown it is clear that the epidemic routing random walk model had decreased benchmark in delivering the messages as was increased the speed of the nodes. In the case of the shortest path map based model, as the speed increased, the DP increased up to 0.28 (maximum value) and after that, the value of DP remained constant. This is due to the reason that this movement model follows a certain pre 
defined specific path and, therefore, after a certain time, the DP value becomes constant. On the other hand, the random waypoint model had better delivery probability due to the randomness of the movement because there is a good chance of meeting the destination nodes in this case.

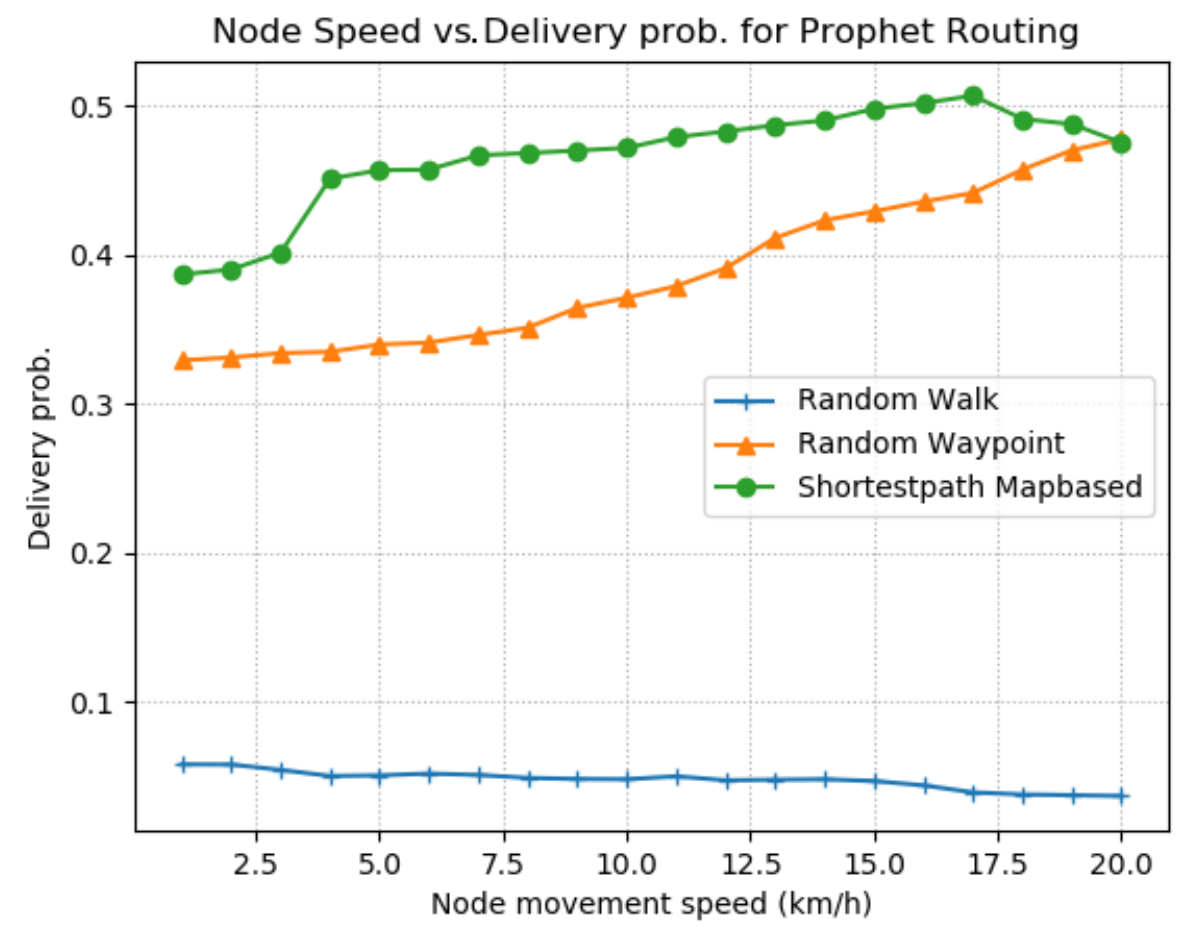

Fig 3. Node speed vs. Delivery Probability: Prophet routing for the proposed Random Walk, Random Waypoint, and Shortest path Map based movement models.

Since Prophet was specifically designed for restricted flooding, which is optimized too, the delivery probability for Shortest path map based and Random waypoint reached up-to 0.48 and 0.50 , respectively, which had successfully achieved the higher benchmark of the delivery probability for Prophet in compare to epidemic, as shown in Figure 3. A sharp fall of DP in case of node speed of up to $20 \mathrm{kmph}$ for shortest path map based model was also observed (from 0.50 up to 0.47 ). This was, perhaps, due to the same reason of the epidemic routing case.

In the case of Spray and Wait, it could be observed a better performance in comparison to Prophet and Epidemic strategy for the shortest path map based mobility (up to a delivery probability $>0.65$ ). The random waypoint model performed an average performance (DP of 0.55), as can be observed in Figure 4. 


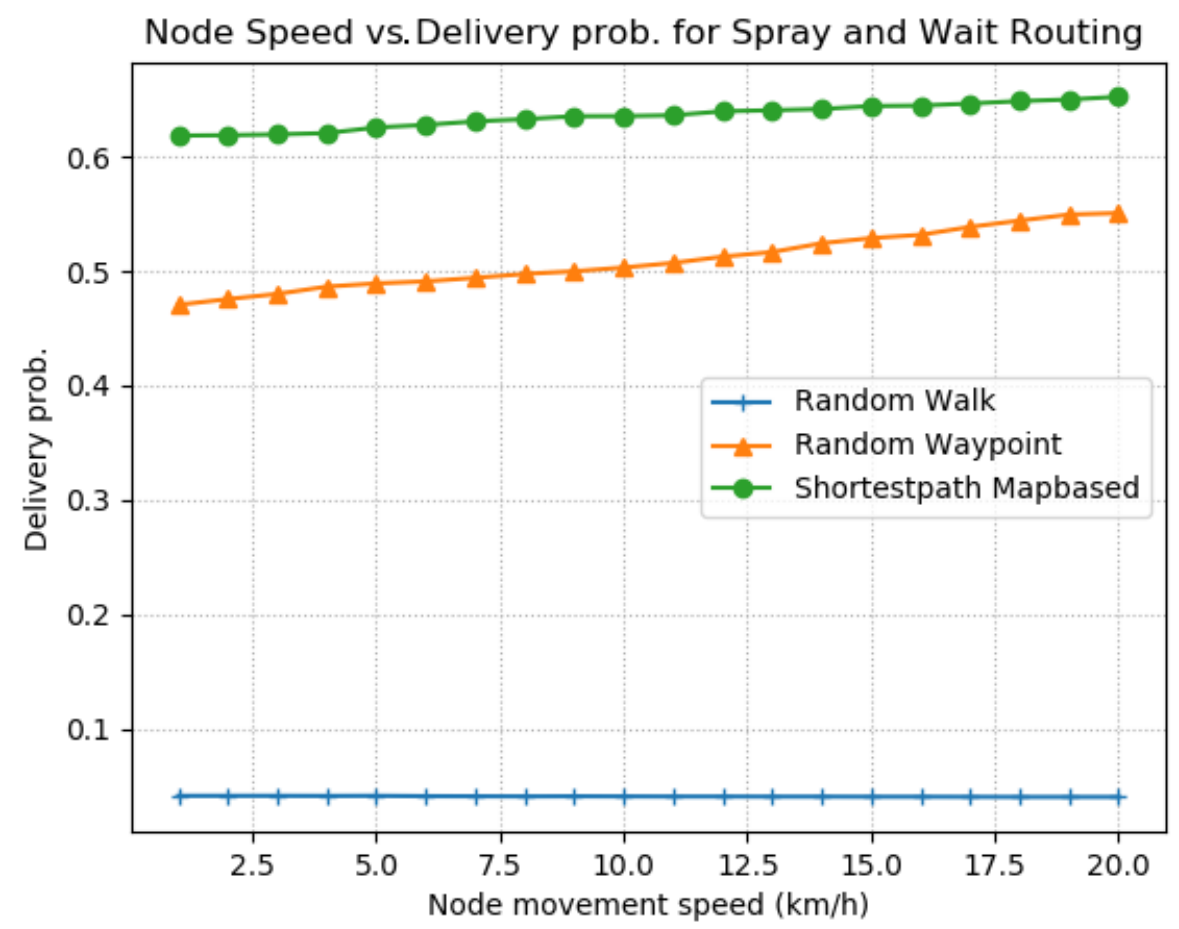

Fig 4. Node speed vs. Delivery Probability: Spray and Wait routing forthe proposed Random Walk, Random Waypoint, and Shortest path Map based movement models.

On the other hand, MaxProp routing produced a greatest delivery ratio, which was equal to 0.8856 for the shortest path map based movement (Figure 5). This was the best performance found amongst all cases, which was due to two main reasons: Firstly, in this case, all the FANET nodes keep track of the messages delivery by using acknowledgments to ensure the delivery. Secondly, during the routing under well organized shortest path map based mobility, choosing an optimal path amongst all possible paths is pretty much effortless. In an organized path, the delivery likelihood with the peer node is quite high. Even if the hop count crosses the threshold value, there still a good chance to deliver the message because of the high delivery likelihood. 


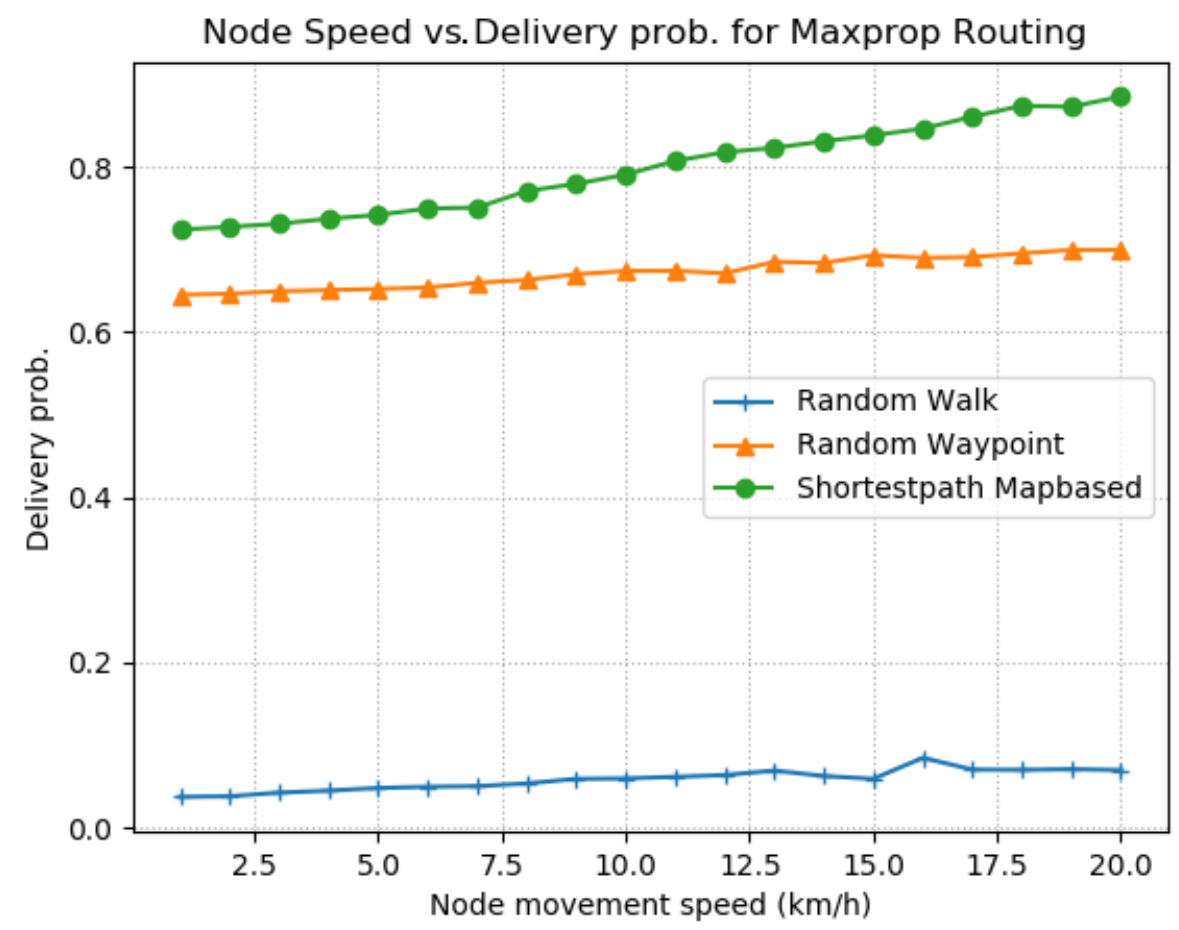

Fig 5. Node speed vs. Delivery Probability: MaxProp routing for the proposed Random Walk, Random Waypoint, and Shortest path Map based movement models.

\subsubsection{Performance of node density vs. delivery probability}

The impact of node density change for the specific routing strategies under the proposed modified mobility scheme can be observed in Figure 6, 7, 8 and 9, respectively. From these figures, it can be realized that the increasing order of node density implied a negative impact on the messages delivery for most of the routing protocol models. For Epidemic routing strategy, the Random waypoint and shortest path map based mobility produced almost the same results. This was because under both of these mobility patterns, the message copy generated by Epidemic is almost the same and, as the number of node increases, more redundant message copies are generated causing, therefore, the exhausting of the messages buffer and the drop of messages. In such situation, the rate of messages drop is higher than the rate of delivered messages, which leads to a performance decrease. 


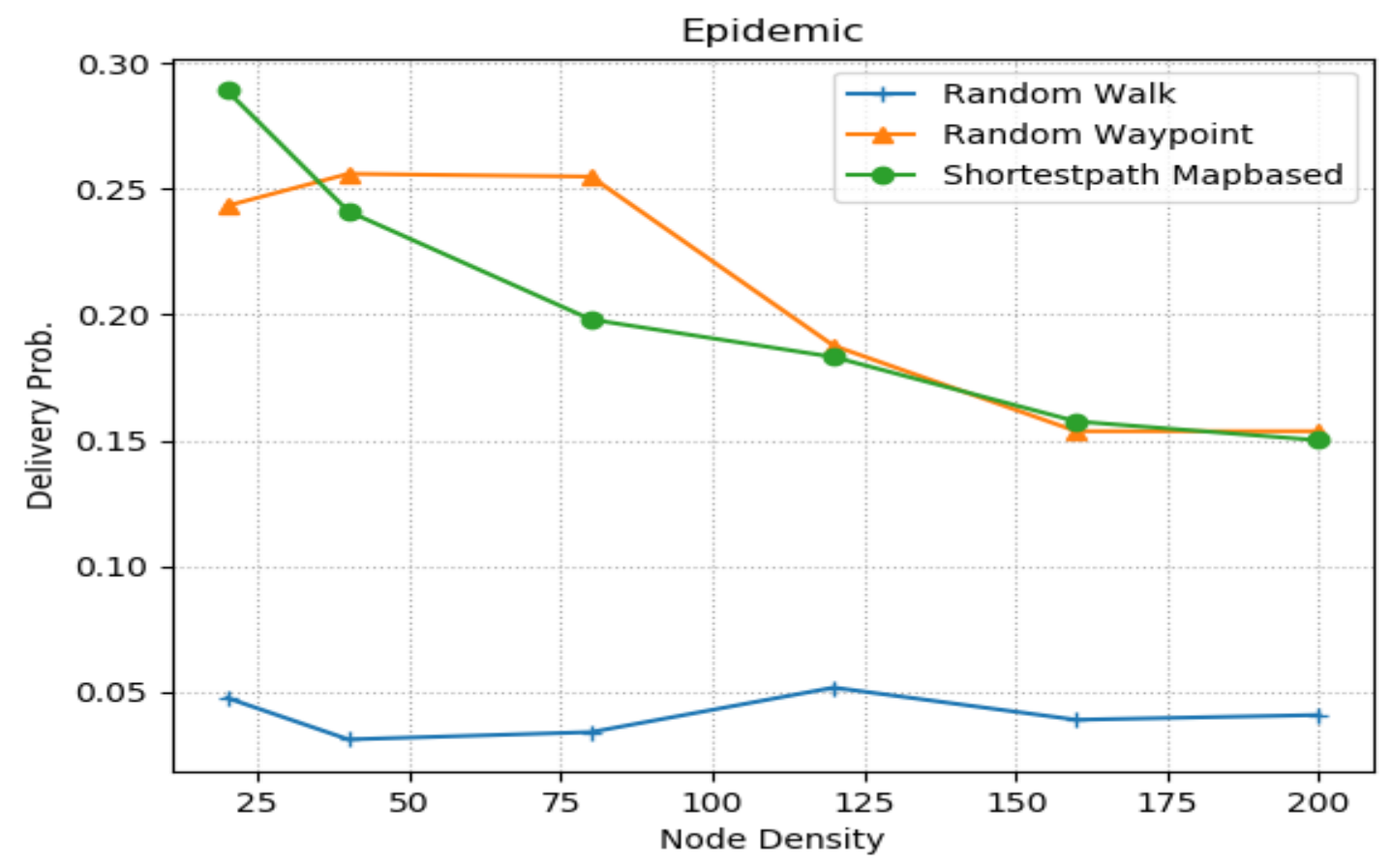

Fig 6. Node density vs. delivery probability: Epidemic routing for the proposed Random Walk, Random Waypoint, and Shortest path Map based movement models.

In the case of Prophet routing, due to the restricted flooding nature, the decrement of delivery probability was not drastic but diminishing instead, Figure 7. In this case, shortest path map based model obtained a relatively better and predictable performance relatively to random waypoint model, and the rate of change of delivery probability was found to be the lowest one.

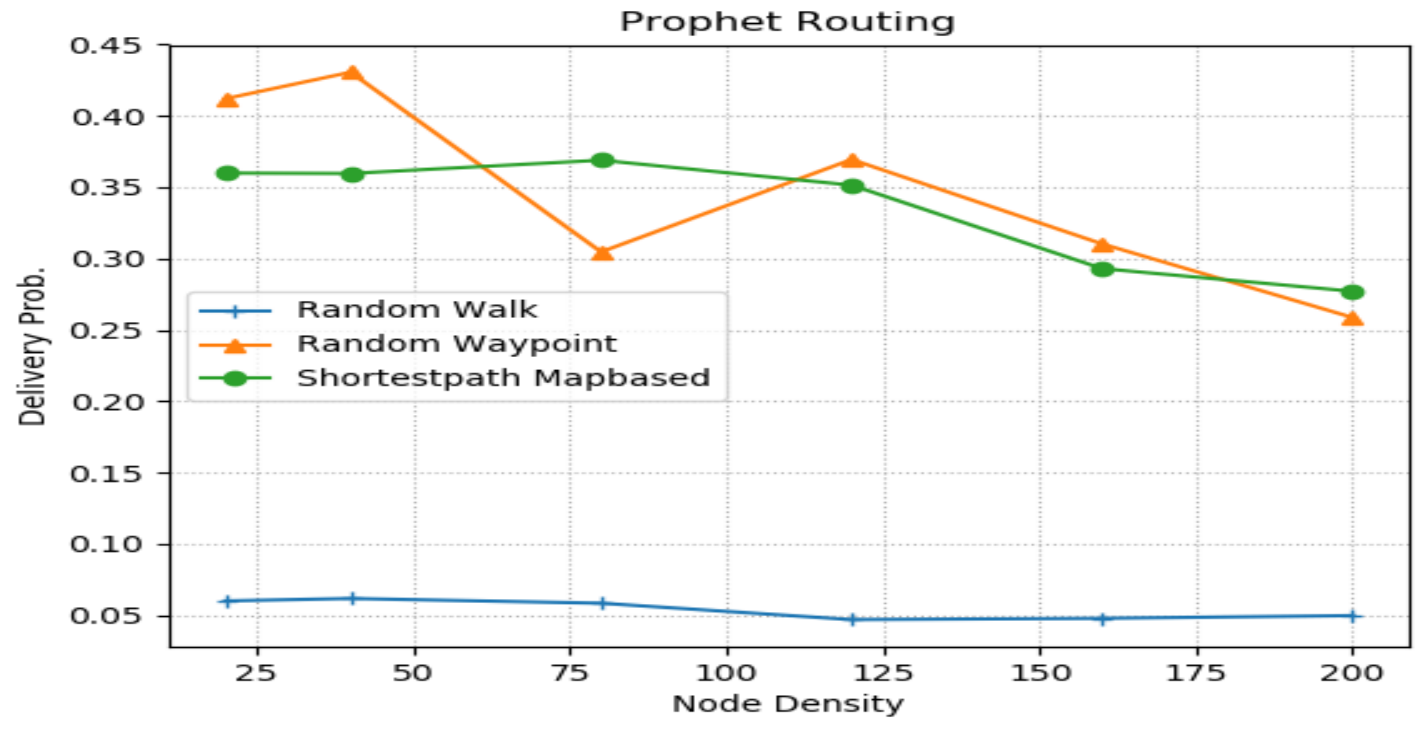

Fig 7. Node density vs. delivery probability: Prophet routing for the proposed Random Walk, Random Waypoint, and Shortest path Map based movement models. 
On the other hand, spray and wait strategy performed better than the other techniques under study (Figure 8). In this case, the decrement of Delivery probability was insignificant for the shortest path map based mobility, and, as we incremented the node density from 40 to 80, the delivery probability increased drastically. In spray and wait, the fall of Delivery probability was significantly low due to basic phenomena of the protocol itself. As it is common accepted, in spray phase, if the destination is not found, then each node contains the messages copy and makes a direct contact in wait phase. It means that the spray and wait protocol depends on direct contact at wait stage. In this scenario, if the number of node increases, the chance of direct contact also increases. Although buffer overhead increases, that has effect on the delivery performance due to the direct contact message, and therefore the delivery probability improved, which limited here the maximum benchmark for messages delivery under shortest path map based mobility of up to $60 \%$ with a 200 nodes density.

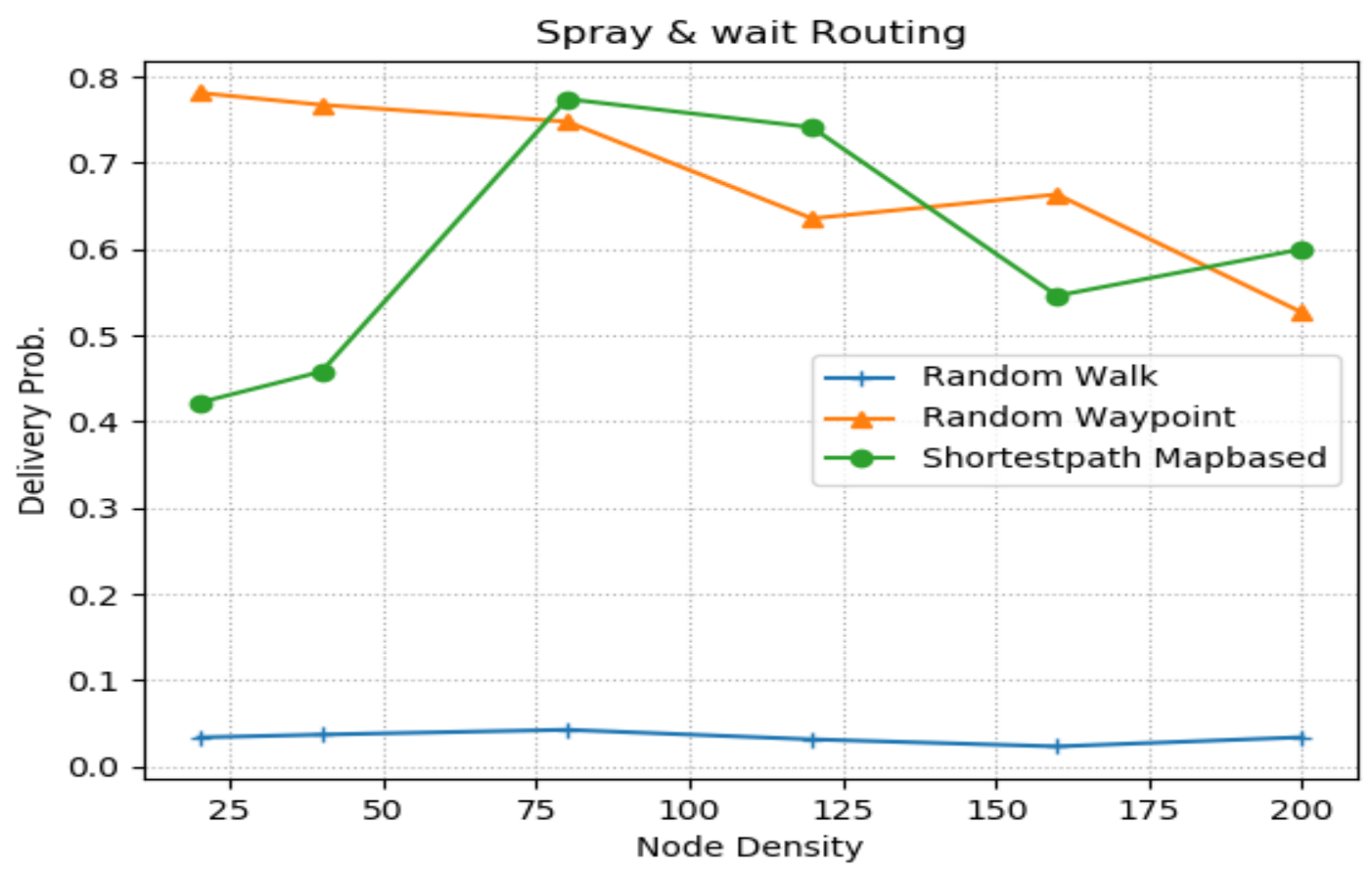

Fig 8. Node density vs. delivery probability: Spray and wait routing for the proposed Random Walk, Random Waypoint, and Shortest path Map based movement models.

On the other hand, MaxProp routing gave a minimum delivery probability of 0.41 and 0.29 for shortest path map based and random waypoint mobility, respectively. In the case of the number of nodes between 40 and 80, a sharp rise in delivery probability was observed. However, the random walk model produced a minimum benchmark in all studied cases.

By considering the above performance results, it can be concluded that, for an increasing node density, the Spray \& Wait routing strategy with shortest path map based mobility performs best in a Flying Adhoc Network scenario due to a high chance of direct contact.

The reason of the fall of delivery probability found in most of the cases is that, in normal cases when node density increases, more replicated messages will also be generated. As more messages are generated, more message exchange occurs, which results in buffer overflows. In normal situations, message TTL has a large value, so the rate of packet generation is mostly higher than the rate of packet drop or delivery; 
therefore, congestion is obvious. That is way in most routing situations, DP decreased drastically as the number of node increased.

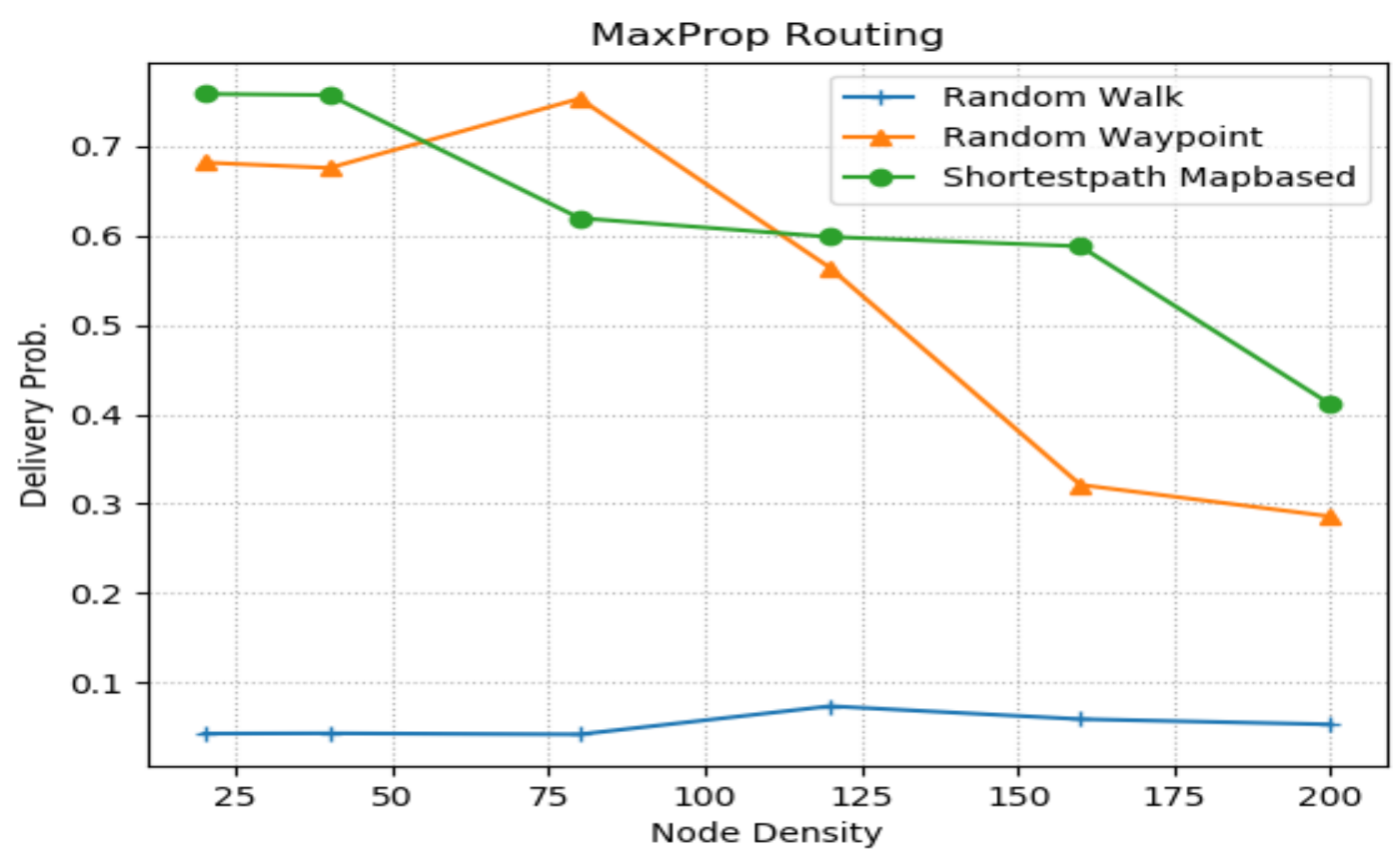

Fig 9. Node density vs. delivery probability: MaxProp routing for the proposed Random Walk, Random Waypoint, and Shortest path Map based movement models.

\subsubsection{Performance evaluation based on overhead ratio, average latency and buffer time}

The message overhead ratio obtained for each different routing strategy is illustrated in Figure 10. From the graphs shown, it is apparent that Spray and waits obtained the minimum message overhead. This has even set a minimum benchmark for the shortest path map based model. In the case of epidemic routing, the overhead was comparatively high because of the unrestricted nature of message relay and the massive generation of message replica. In the case of Prophet protocol in Shortest path map based model, the message overhead had increased because this strategy tries to achieve a high delivery probability with a varying speed, as the Density probability suddenly falls down after the node speed of $17.5 \mathrm{kmph}$ and, therefore, the overhead increased exorbitantly. For MaxProp Strategy, the message overhead for random waypoint and shortest path map based mobility was quite high in comparison to Prophet because of the generation and buffering of both messages that are under threshold and beyond threshold values. In case of random walk, as the chances of delivery become significantly low, more messages got, therefore, buffered relayed to next hop, but they were hardly delivered, which resulted a high overhead ratio. 


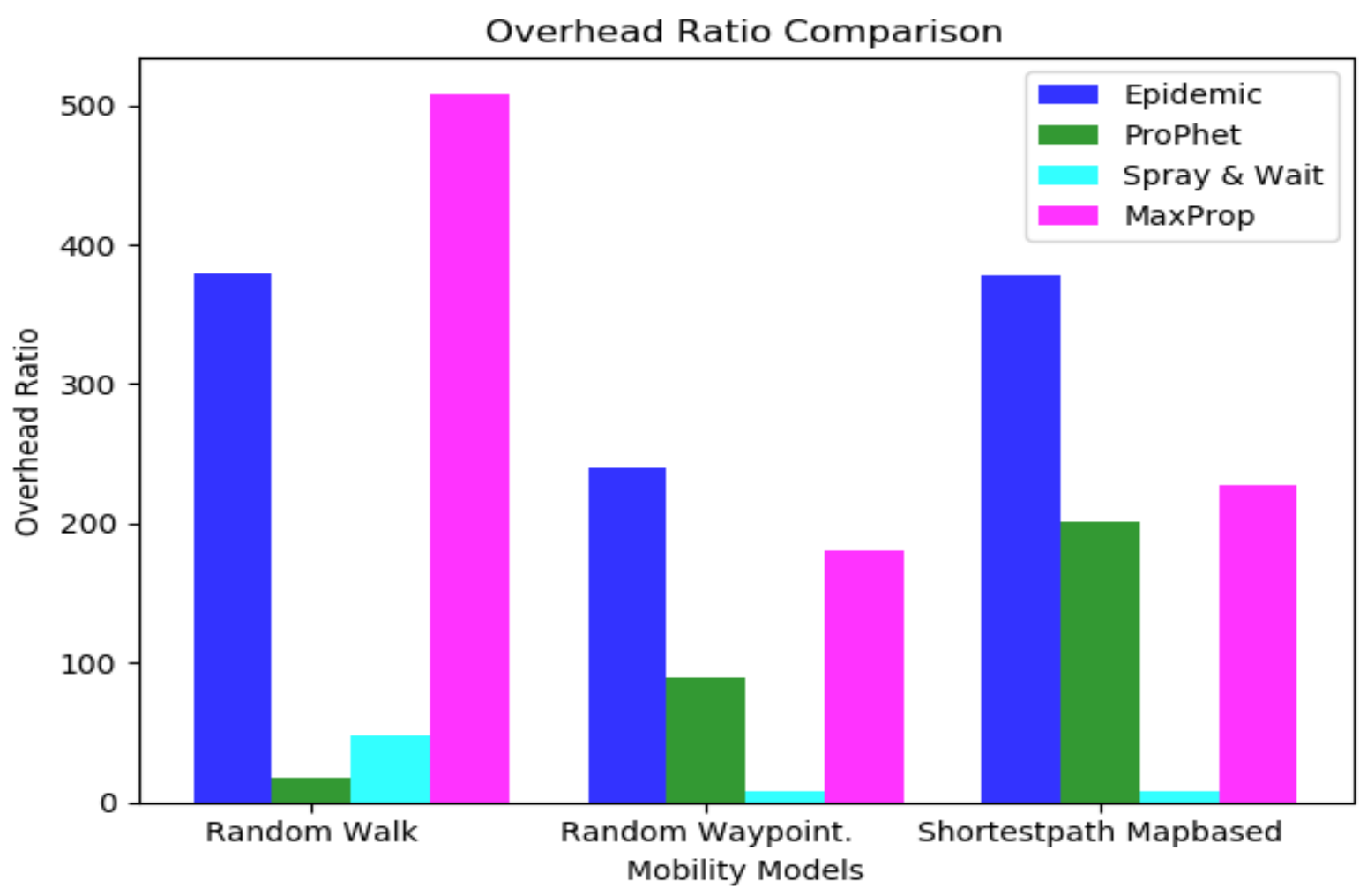

Fig 10. Comparison of message overhead ratio for the four different routing strategies under the proposed mobility models.

Figure11 illustrates the characteristics of the message delivery latency of each routing strategy on the proposed mobility scenario. For a constant buffer size, the average latency for random walk model was always beyond 1400, except for spray and wait. The shortest path map based model performed a consistent latency average for all routing strategy because of its better predictability of the navigation path of the FANET nodes. It is guaranteed that a relatively better average latency of 20-150 units, a node speed between 5 to 15 kilometers per hour is achievable by FANET nodes in an ideal condition. Also, the hop count value directly affected the average latency, as the hop count increased the chances to deliver the data to the destination also increased because the message moves from the local buffer and migrates throughout the innumerable nodes of the network. MaxProp suffered from high latency in case of random walk and random waypoint because of the inherent property of the protocol to buffer and forward the messages according to hop count for packets under threshold, and also based on likelihood for beyond threshold packets. 


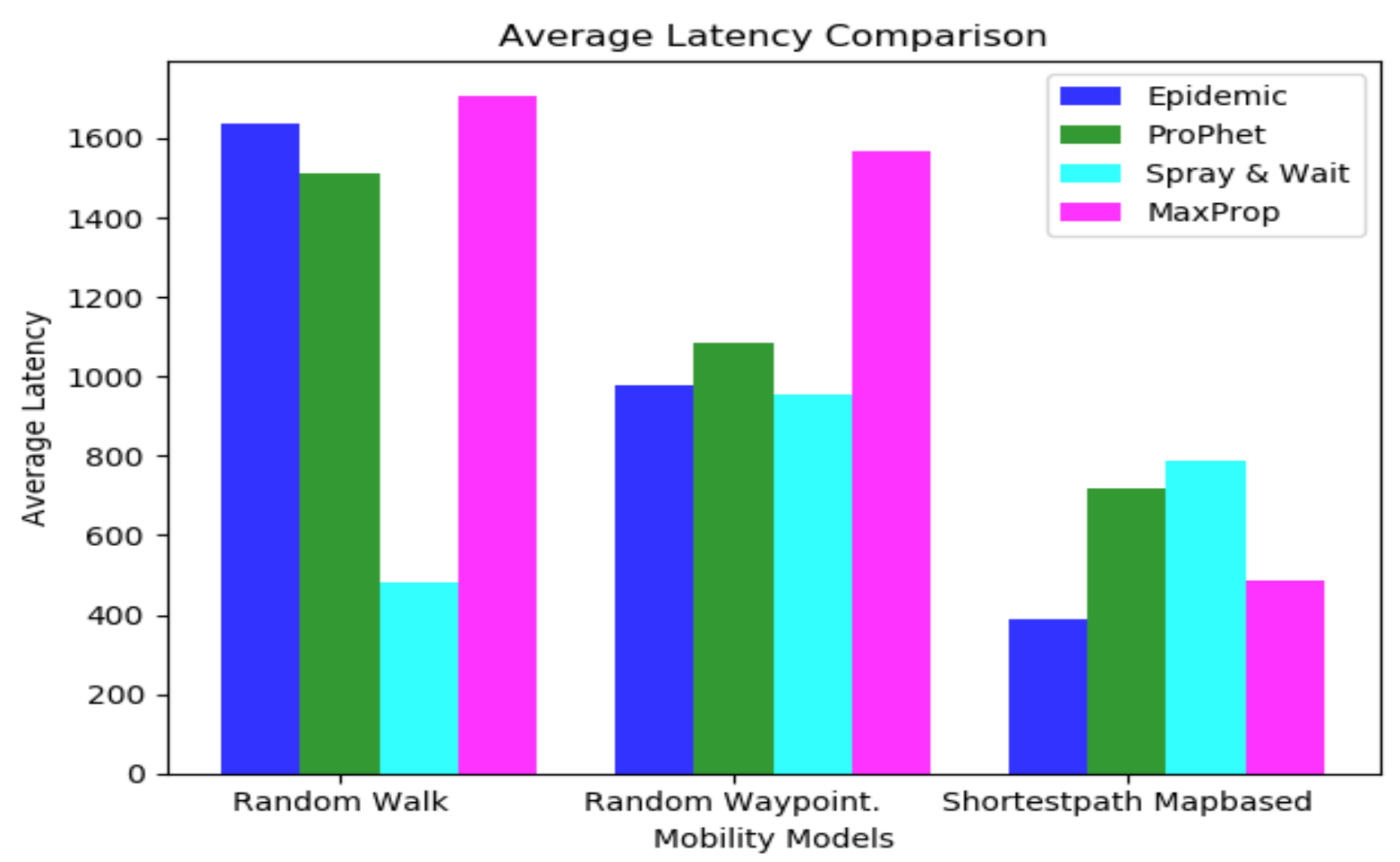

Fig 11. Comparison of Average message delivery latency for the four different routing strategy under proposed mobility models.

The buffer time attribute shown in Figure 12 depicts the results obtained as to the duration of the retention of the message $i$ in the buffer. The buffer time parameter plays an important role to figure out the utilization of the buffer memory and hence the delivery probability. A high message buffer time implies mostly an increasing delivery probability as the message stored into the buffer has a better chance to be forwarded. There is a trade-off between buffer time and mobility pattern. A mobility that does not perform well due to high node speed may significantly reduce the delivery probability, although having a high average buffer time. In the case of Random walk model, this phenomenon was significantly perceptible. On the other hand, in the case of spray and wait, the high buffer time resulted in a good delivery probability. For Prophet, this phenomenon worked in an inverse manner due to its restricted flooding nature. However, in the case of MaxProp, the behavior found was different. Since MaxProp works on the basis of delivery likelihood of the peer, the messages that are generated mostly relay on their priority values. On the other hand, packet shaving low priorities are mostly deleted from the buffer. Therefore, no messages stay a long time in the buffer. 


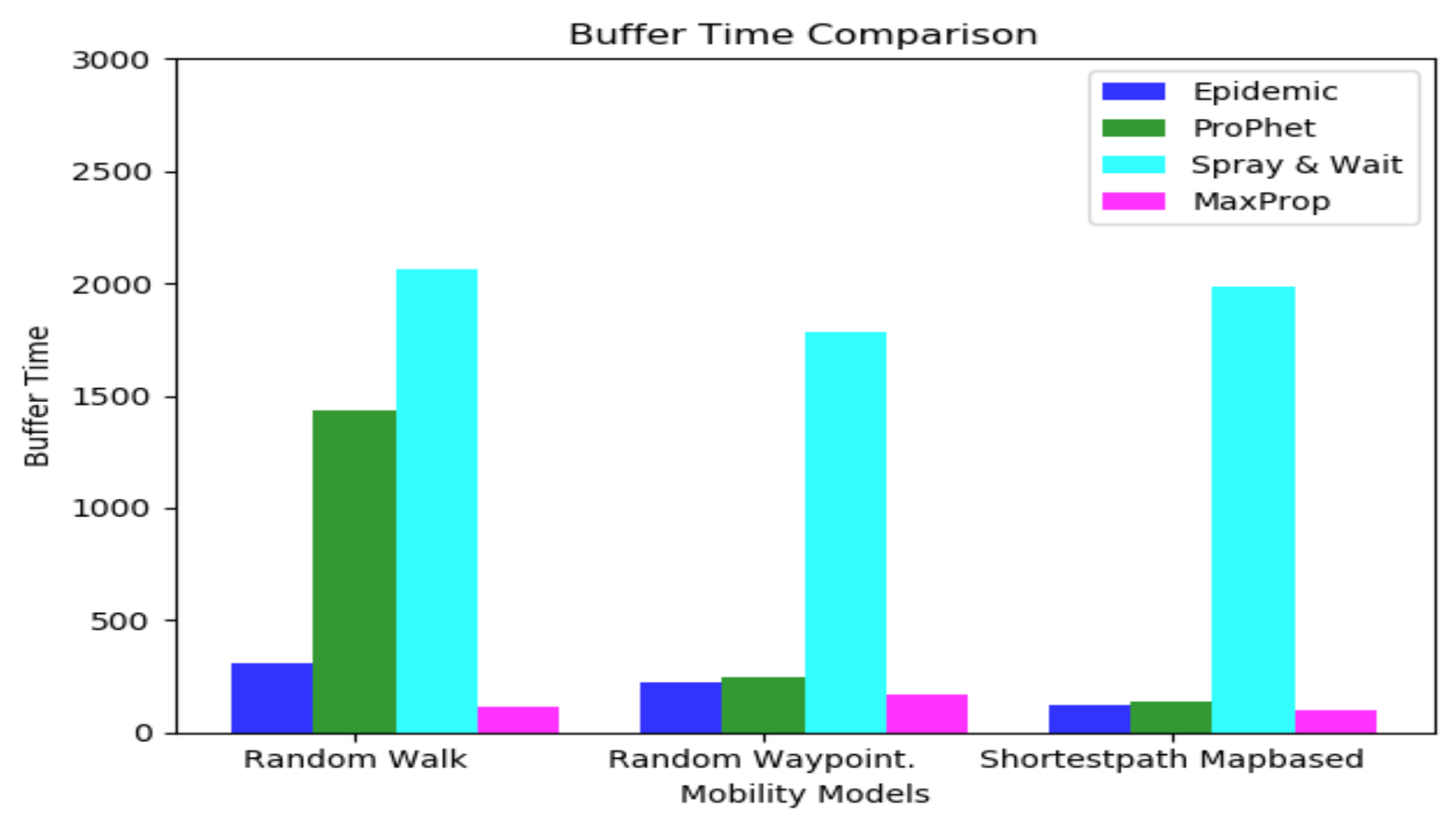

Fig 12. Comparison of Average Buffer time for different routing strategies under proposed mobility models.

The next crucial parameter that was considered in this work was the relation between the delivery probabilities with respect to the buffer size. It was found that the delivery probability dramatically increased for all routing algorithms regardless of the used mobility model. This was found because a large number of buffers imply higher messages storing capability in the nodes, hence less chance of packet drop. In the case of latency, the enhancement of the buffer size plays a negative role and, consequently, it increases the latency. 


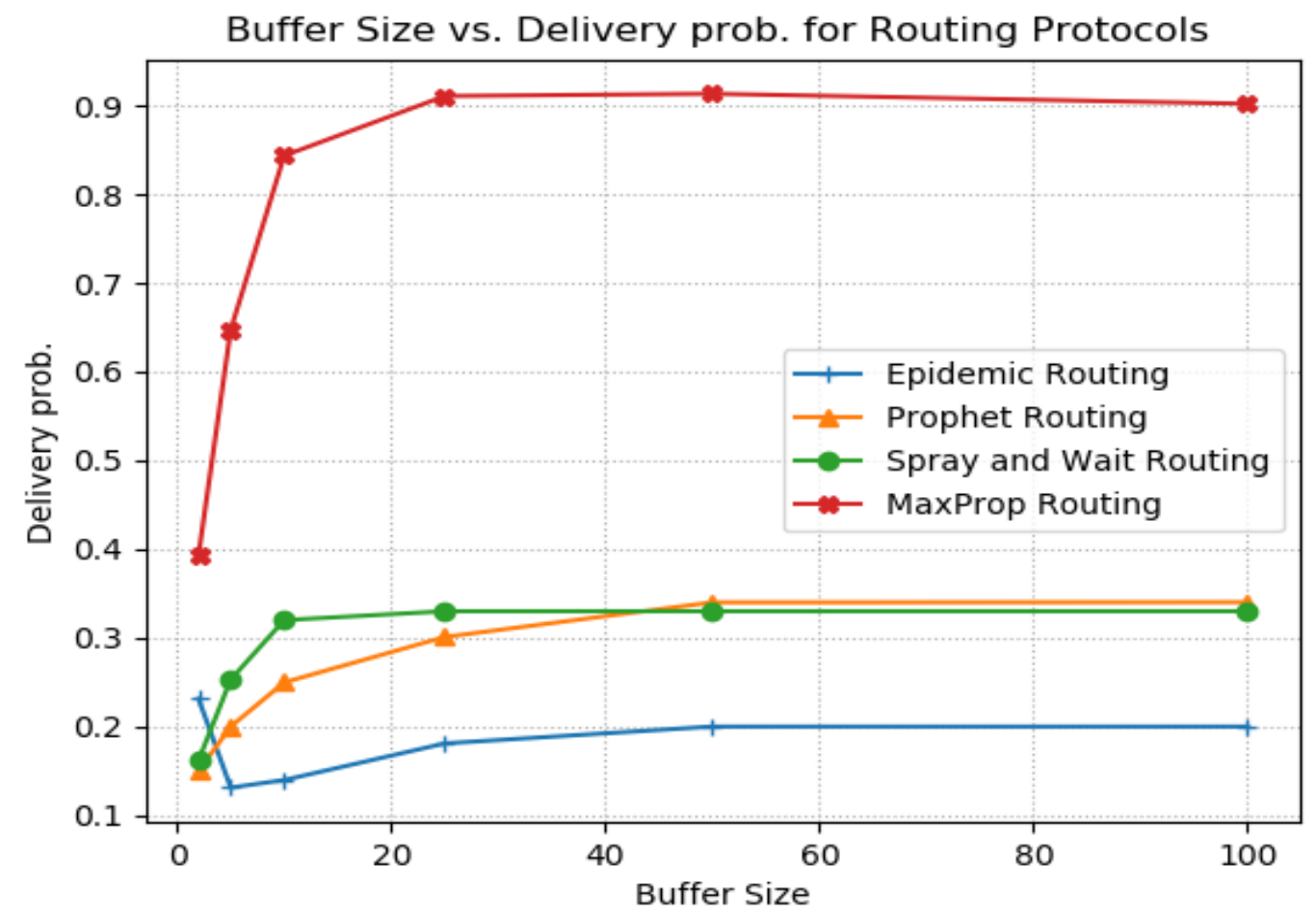

(a)

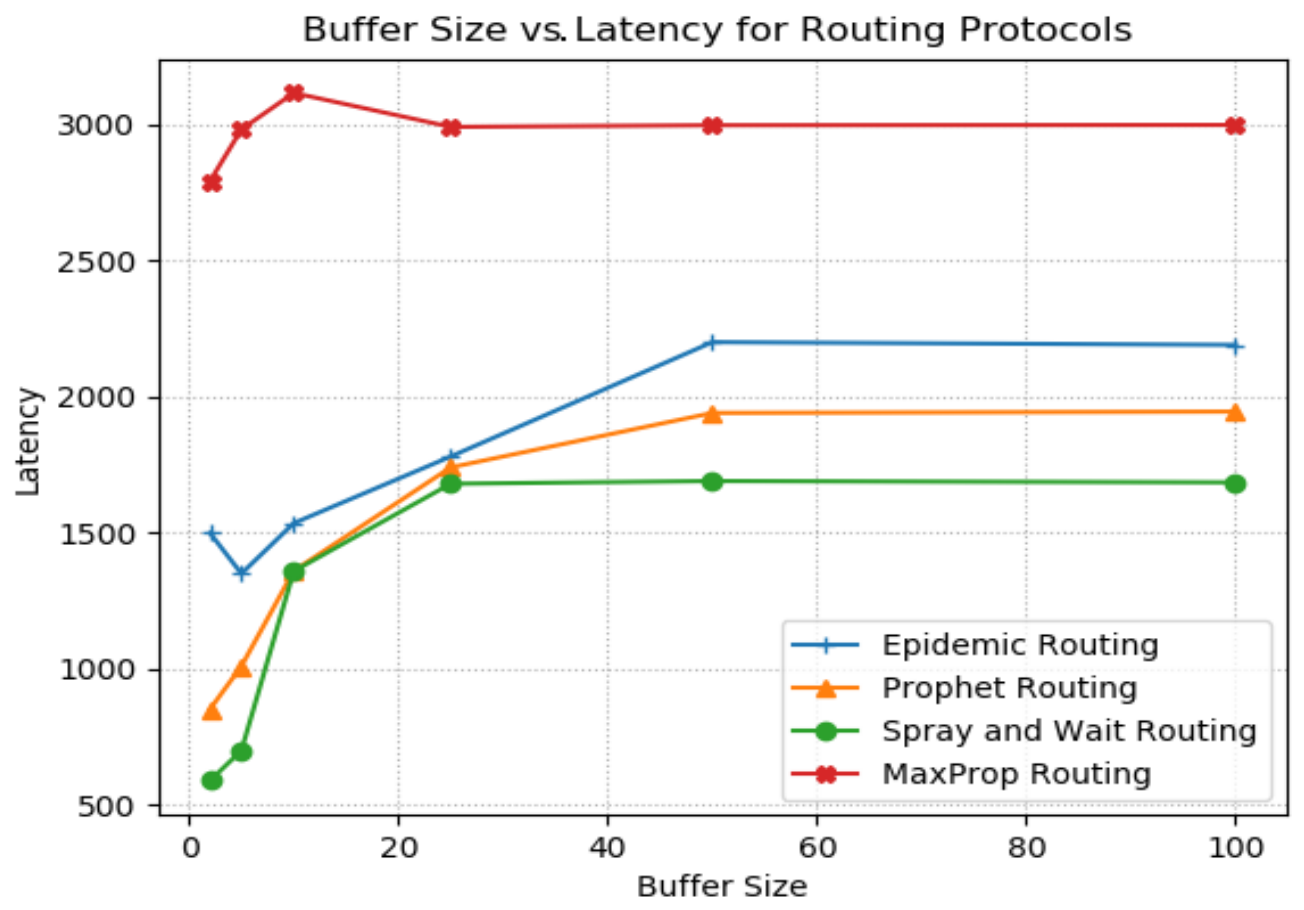

(b)

Fig 13. (a) Delivery Probability vs. Buffer size and (b) Latency vs. Buffer Size for the four routing strategies under study. 
Figure 13(a) shows the effects of the increment of the buffer size on the delivery probability. From the graph shown, it is clear that the MaxProp outperforms amongst all as the buffer size increased to 20MB or more. In the case of Epidemic, the buffer size increased within the range of 30MB. In Prophet, the delivery probability crossed Epidemic as increased the buffer size beyond 40MB. Spray and wait obtained the lowest benchmark as to the maximum delivery probability, which achieved an upper limit of 0.2. Therefore, it can be concluded that anode with high buffer size does not perform well in the case of the spray and wait due to its wait phase. Secondly, in the case of MaxProp, high buffer size increased the chance of delivery rate because more prioritize messages and acknowledgments had been buffered and relayed towards destination. Additionally, it was observed that under the latency point of view, MaxProp suffered more than the other three approaches, Figure13 (b). As discussed earlier, prioritized messages buffered and relayed based on the threshold level of hop count increases the chances of latency. In this case, spray and wait performed with an unaffected behavior since the buffer size does not matter for increasing delivery latency. However, Prophet, in this case, accomplished an average performance. In order to achieve the best performance in the Flying Ad-hoc Network scenario, shortest path map based movement is supposed to perform better than the other two models. Most of the routing strategies perform well under this mobility model. Spray and wait routing with shortest path map based mobility is perhaps the optimum methodology by looking at the performance point of view because, except one or two cases, it out performed others in all studied cases.

\subsubsection{Performance comparison of modified mobility models}

Figure 14 depicts the average message delivery performance found using all studied routing strategies with both standard and modified mobility models.DTN standard routing strategies were primarily designed for message transfer amongst pedestrians, ground vehicles and stationary nodes, which are supposed to be at the ground level with a maximum altitude up to 2 to 5 meters. As we are extending the DTN communication philosophy for flying nodes, it is obvious that there may be some altitude difference between nodes in some specific scenario. In that case, the airborne nodes my fly at an altitude of hundred to thousand meters and difference may vary from 5 to 100 meters approximately. Considering this situation, we developed a comparison in terms of average message delivery performance of standard and proposed mobility models for all studied routing strategies. From Figure 14, it is clear that message delivery ratio achieved by shortest path map based model was the best one among all strategies, and it is also shown that the modified mobility models out performs the standard mobility models generally considered for DTN mobility of ground nodes. Since the change of altitude is a primary factor in case of all the models, and the nodes move freely in the 3D space, the performance of the modified mobility model was superior in comparison to the traditional random walk, random waypoint and shortest path map based model. 
Avarage delivery prob. for all routing achived by mobility models.

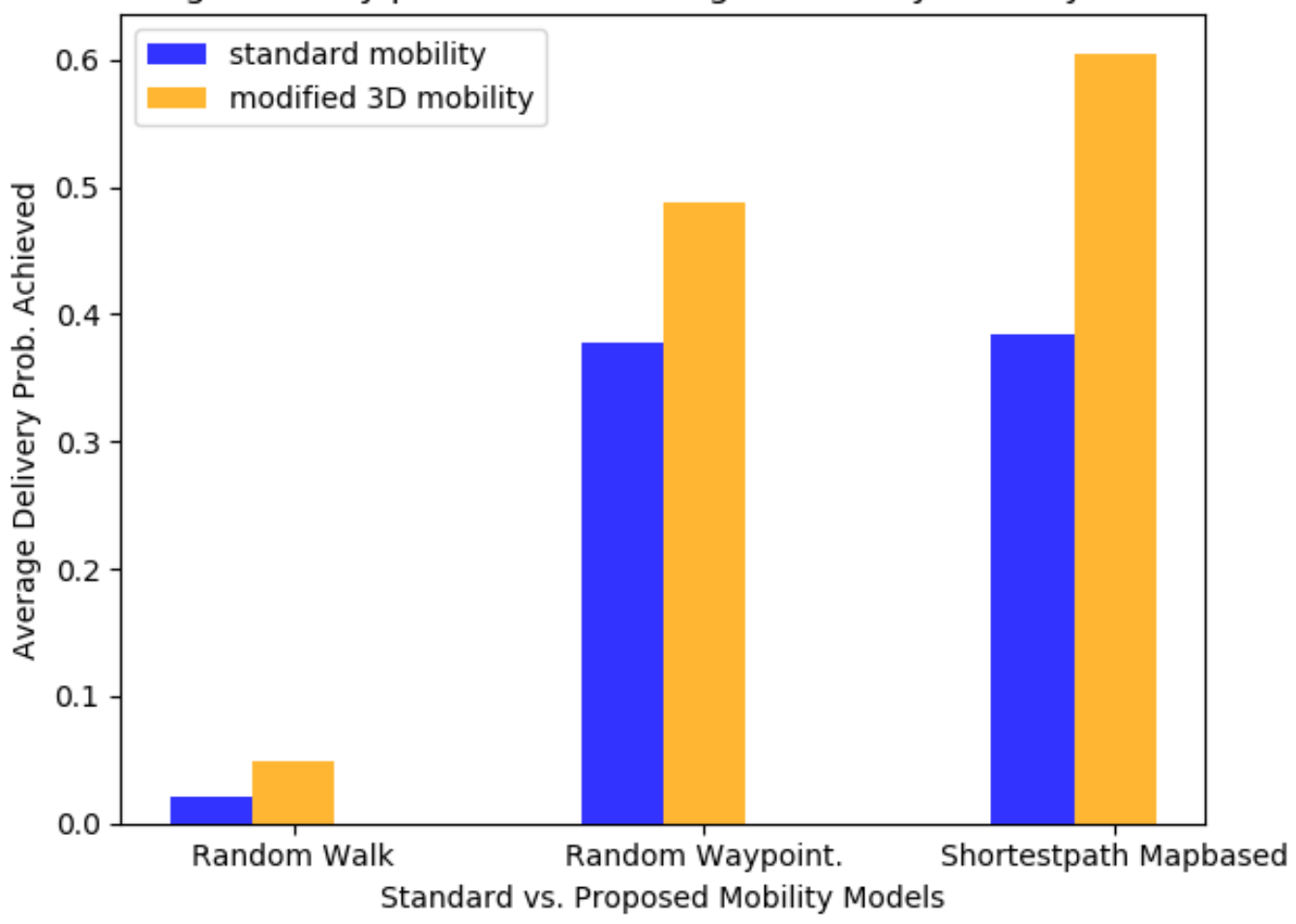

Fig 14. Message delivery prob. Comparison of standard and modified mobility models.

\section{Energy Analysis}

The measurement of the energy dissipation was done based on several assumptions. Each FANET node was considered an onboard transmission and receiving unit. The nodes had IEEE802.11 interface with 100 -meter transmission range. We considered the total power of the network as $520000 \mathrm{mAh}$ at the starting of the simulation. The other used parameters are indicated in Table 3. Here, initial energy is the energy hold by all nodes within the network at time $\mathrm{T}=0$. The absolute energy dissipation is the parameter that reflects energy dissipated within an instant of time by the entire network. Scan energy implies the energy dissipated to scan the peer nodes, and the transmission energy is the amount of energy dissipated to transmit and receive the message packets.

Table 3: Used Simulation Parameter Settings for Energy.

\begin{tabular}{|l|l|}
\hline \multicolumn{1}{|c|}{ Variable } & \multicolumn{1}{c|}{ Value } \\
\hline Total Power & $520000 \mathrm{mAh}$ \\
\hline Initial Energy & $6.17 \times 10^{6} \mathrm{~J}$ \\
\hline Scan Energy & $0.014 \mathrm{~J} / \mathrm{s}$ \\
\hline
\end{tabular}




\begin{tabular}{|l|l|}
\hline Scan Interval & $0.5 \mathrm{sec}$ \\
\hline Transmission Energy & $0.13365 \mathrm{~J} / \mathrm{s}$ \\
\hline
\end{tabular}

The energy dissipation found for the individual routing algorithms under study is illustrated in Figure 15. Here, we considered the average energy dissipation for the well-known routing strategy under all three mobility model with respect to time. From Figure 15, it is clear that the energy dissipation for MaxProp routing was significantly high, and that it increased drastically after 10 hours of simulation. From Figure 15, it can be also realized that MaxProp routing outperforms all the others in terms of delivery probability. One can also say the shortest path map based model had the best performance of MaxProp under high node density as well as high speed.

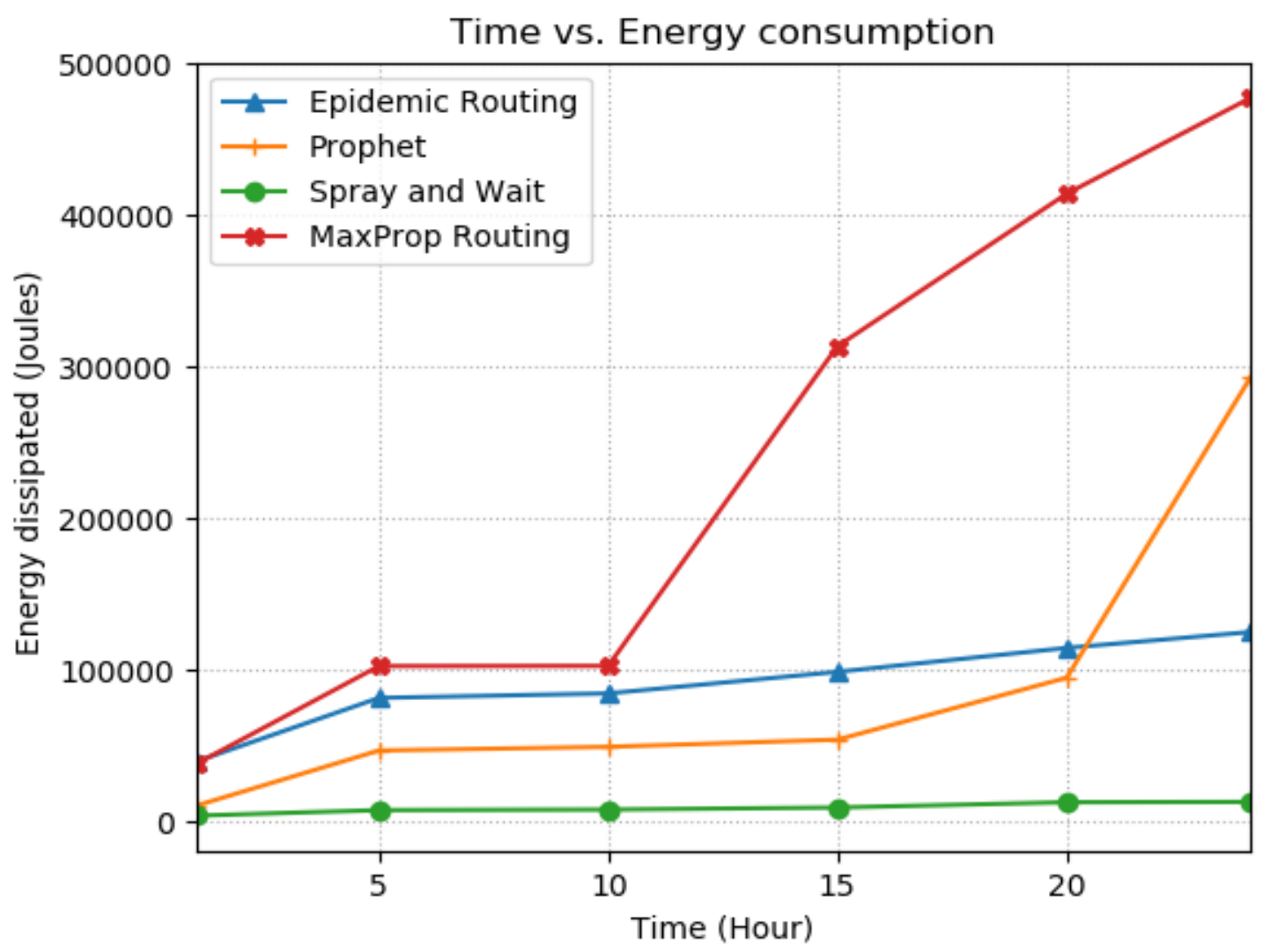

Fig 15. Energy consumption w.r.t time for the routing algorithms.

Figure 16 illustrates the overall power consumption by the network with respect to the average delivery probability achieved by a routing protocol under specific mobility model. From this figure, it can be understood that shortest path map based mobility model achieved the best delivery probability relative to the other two mobility models. This guarantees an optimum performance of all routing protocols under the shortest path map based mobility model. 


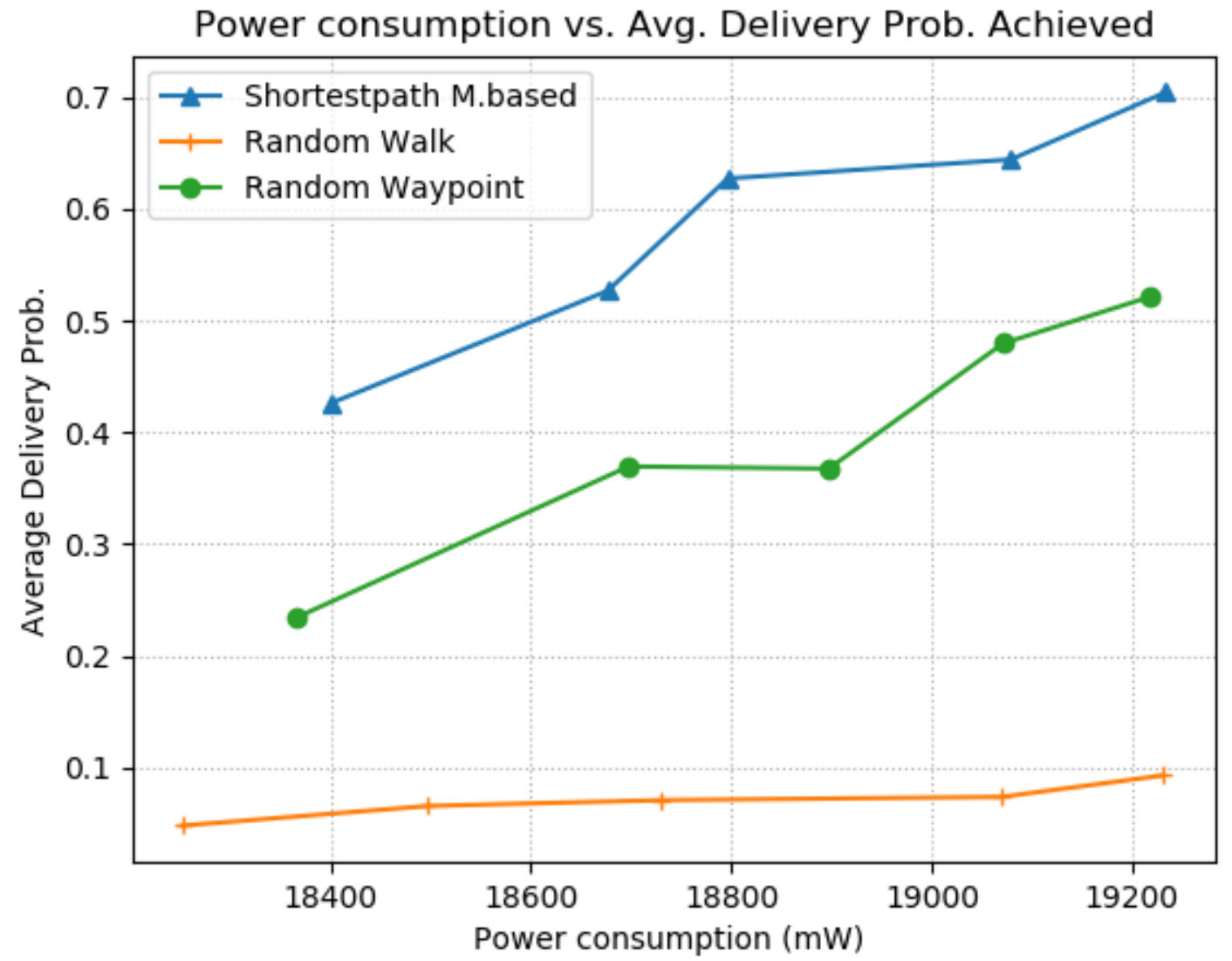

Fig 16. Power Dissipation vs. delivery probability achieved by the proposed mobility model.

\section{Conclusion}

In this work, the DTN assisted FANET scenario, which is noticeably relevant for the tactical application of Flying Ad-Hoc networks, was studied. The study of the newly established mobility models with the standard routing strategy gives a boulevard to implement Flying Ad-Hoc networks in intermittently connected and challenged scenarios. The different routing protocols and their behavior were analyzed, and it was found that the shortest path map based mobility performs best in the context of delivery probability by considering Prophet and MaxProp routing approaches. Additionally, if an average latency is considered, the same model outperforms the other models for both Prophet and MaxProp approaches. Further, under the buffer size vs. delivery probability standpoint, the MaxProp had the best results amongst the competitors. However, in an increasing node density, spray and wait as well as MaxProp perform well. In an energy-based perspective, it can be claimed that the shortest path map based model is most suitable for FANET scenario. Therefore, in a nutshell, we can conclude that MaxProp routing protocol under shortest path map based mobility gives optimal performance in Flying Ad-hoc networks. The performance study of different frameworks gives a way forward to choose the proper network parameters to get an upstanding result in terms of performance. In order to improve the performance of the routing methodology, local optimal buffer management with precise packet dropping policy can be introduced. Messages queue management is also necessary to achieve better performance in case of 
epidemic, and Prophet. We can assign the rank or priority of messages that are buffered. Based on the rank, either the messages are delivered or dropped. To increase the chance of delivery of the message for Prophet, the delivery predictability can be tuned using a modified fuzzy Prophet Mechanism [50]. In this case, the range of delivery predictability has to be chosen based on the boundary value $\{0,1\}$. If the value is found lower than 0.5 , then it is associated with low delivery predictability, and for greater than 0.5 , too high delivery predictability. The deliver predictability can be found using a membership function where high membership is considered as a better forwarder. The routing methodology used was designed for the DTN; hence, further exploration and the reconstruction of the hybrid mobility and the routing are considered to be the future research challenges. Secondly, the performance of mobility can be tuned by introducing the following scheme. Scale-free mobility regimes [53] can be introduced for the random walk mobility model to tune up its performance. The scheme is executed in two phases. In the first phase, an initialization has been done where each node is arbitrary distributed in a geographical location. In the movement phase, if a node is willing to move to the next waypoint with a probability of $p\left(\dot{\eta}_{l}\right)$, then it is selected the next waypoint with a probability of $p\left(\mu_{i}\right)$ based on the priori sample computed from $F\left(\omega_{v}\right)$. Then, it moves to the next waypoint with random speed $\left(\mathrm{v}_{\min }, \mathrm{v}_{\max }\right)$. Using this methodology, the density distribution is highly close to real-world trace having a confidence interval of about $95 \%$. In order to achieve real-time prediction of mobility, a Spatio-Tempo-SocioSemantic-Aware model can be used [54]. This model classifies the frequently visited location in the factor of time. The main component of the model is a location sphere that comprises the frequently visited waypoints that are visited with often and some non-frequent waypoints. By segregating the frequently visited waypoints to non- frequently visited waypoints, the performance can be improved. A neural learning machine based model [55] can be taken into account based on multilayer perceptron and Extreme Learning Machine techniques. This solution can achieve an MSE score within $10^{-8}$ to $10^{-5}$ if a 6 neuron layered configuration is used. The flying Ad-Hoc network in a DTN perspective also imbibes the energy analysis and optimization, which is another acclaimed research domain.

In our belief, this work opens the potential research direction for DTN assisted FANET design and their application for smart city Internet framework design, environmental monitoring, and others. The application is not limited in the Earth, but also in extra-terrestrial research, like an exploration of life in the neighbor planets like Mars.

\section{References}

1. Mahony, Robert, Vijay Kumar, and Peter Corke. "Multirotor aerial vehicles." IEEE Robotics and Automation magazine 20, no. 32 (2012).

2. NilanjanDey, and Amartya Mukherjee. Embedded Systems and Robotics with Open Source Tools. CRC Press, 2016.

3. Bekmezci, Ilker, OzgurKoraySahingoz, and ŞamilTemel. "Flying ad-hoc networks (FANETs): A survey." Ad Hoc Networks 11, no. 3 (2013): 1254-1270.

4. Kumari, Kanta, Basant Sah, and Sunil Makar. "A survey: different mobility model for FANET." International Journal of Advanced Research in Computer Science and Software Engineering 5, no. 6 (2015). 
5. Sahingoz, OzgurKoray. "Networking models in flying ad-hoc networks (FANETs): Concepts and challenges." Journal of Intelligent \& Robotic Systems 74, no. 1-2 (2014): 513-527.

6. Zheng, Zhigao, Arun Kumar Sangaiah, and Tao Wang. "Adaptive communication protocols in flying ad hoc network." IEEE Communications Magazine 56, no. 1 (2018): 136-142.

7. Lewis, M., F. Templin, B. Bellur, and R. Ogier. "Topology broadcast based on reverse-path forwarding." IEEE MANET Internet Draft (2002).

8. Zhang, Xinming, Xulei Cao, Long Yan, and Dan Sung. "A street-centric opportunistic routing protocol based on link correlation for urban vanets." IEEE Transactions on Mobile Computing 1 (2016): 1-1.

9. Oubbati, Omar Sami, AbderrahmaneLakas, Fen Zhou, Mesut Güneş, and Mohamed BachirYagoubi. "A survey on position-based routing protocols for Flying Ad hoc Networks (FANETs)." Vehicular Communications (2017).

10. Oubbati, Omar Sami, AbderrahmaneLakas, Fen Zhou, Mesut Güneş, NasreddineLagraa, and Mohamed BachirYagoubi. "Intelligent UAV-assisted routing protocol for urban VANETs." Computer Communications 107 (2017): 93-111.

11. Singh, Harpreet, and Prashant Singh Guide Rana. "An Emergency Message Dissemination Protocol Using Greedy Forwarding Technique and Clustering for VANETS." PhD diss., 2017.

12. Cui, Bo, and $\mathrm{Ru}$ Li. "A greedy and neighbor aware data forwarding protocol in named data MANETs." In Ubiquitous and Future Networks (ICUFN), 2017 Ninth International Conference on, pp. 934-939. IEEE, 2017.

13. Maxa, Jean-Aimé, Mohamed-Slim Ben Mahmoud, and Nicolas Larrieu. "Survey on uaanet routing protocols and network security challenges." Ad Hoc \& Sensor Wireless Networks (2017).

14. Khan, Irfan Latif, Riaz Hussain, Adeel Iqbal, Atif Shakeel, Shakeel Alvi, Waseem Abbas, Qadeer ul Hasan, and Shahzad A. Malik. "Design and Evaluation of Self Organizing, Collision Free MAC Protocol for Distributed Cognitive Radio Networks." Wireless Personal Communications 99, no. 2 (2018): 1081-1101.

15. Rosário, Danileno, José Arnaldo Filho, Denis Rosário, AldriSantosy, and Mário Gerla. "A relay placement mechanism based on UAV mobility for satisfactory video transmissions." In Ad Hoc Networking Workshop (Med-Hoc-Net), 2017 16th Annual Mediterranean, pp. 1-8. IEEE, 2017.

16. Patra, Sushovan, Anerudh Balaji, Sujoy Saha, Amartya Mukherjee, and Subrata Nandi. "A qualitative survey on unicast routing algorithms in delay tolerant networks." In Information Technology and Mobile Communication, pp. 291-296. Springer, Berlin, Heidelberg, 2011.

17. Salem, Amer O. Abu, Ghassan Samara, and TareqAlhmiedat. "Performance analysis of dynamic source routing protocol." arXiv preprint arXiv: 1712.04622 (2017). 
18. Han, Guangjie, Lina Zhou, Hao Wang, Wenbo Zhang, and Sammy Chan. "A source location protection protocol based on dynamic routing in WSNs for the Social Internet of Things." Future Generation Computer Systems 82 (2018): 689-697.

19. Bujari, Armir, Claudio Enrico Palazzi, and Daniele Ronzani. "A Comparison of Stateless Positionbased Packet Routing Algorithms for FANETs." IEEE Transactions on Mobile Computing 1 (2018): 1-1.

20. Amjad, Kashif, and Alan J. Stocker. "Impact of node density and mobility on the performance of AODV and DSR in MANETS." In Communication Systems Networks and Digital Signal Processing (CSNDSP), 2010 7th International Symposium on, pp. 61-65. Ieee, 2010.

21. Mukherjee, Amartya, Sayan Chakraborty, Ahmad Taher Azar, Soumya KantiBhattacharyay, Basukinath Chatterjee, and NilanjanDey. "Unmanned aerial system for post disaster identification." In Circuits, Communication, Control and Computing (I4C), 2014 International Conference on, pp. 247-252. IEEE, 2014.

22. Priyan, M. K., and G. Usha Devi. "Energy efficient node selection algorithm based on node performance index and random waypoint mobility model in internet of vehicles." Cluster Computing (2017): 1-15.

23. Deng, Shuiguang, Longtao Huang, Javid Taheri, Jianwei Yin, MengChu Zhou, and Albert Y. Zomaya. "Mobility-aware service composition in mobile communities." IEEE Transactions on Systems, Man, and Cybernetics: Systems 47, no. 3 (2017): 555-568.

24. Ren, Jiajie, Guanglin Zhang, and Demin Li. "Multicast capacity for VANETs with directional antenna and delay constraint under random walk mobility model." IEEE Access 5 (2017): 39583970 .

25. Mukherjee, Amartya, NilanjanDey, Noreen Kausar, Amira S. Ashour, RedhaTaiar, and Aboul Ella Hassanien. "A disaster management specific mobility model for flying ad-hoc network." International Journal of Rough Sets and Data Analysis (IJRSDA) 3, no. 3 (2016): 72-103.

26. S. Hayat, E. Yanmaz, R. Muzaffar, "Survey on Unmanned Aerial Vehicle Networks for Civil Applications: A Communications Viewpoint", IEEE Communications Surveys Tutorials, vol. 18, no. 4, pp. 2624-2661, 2016.

27. He, Ruisi, Bo Ai, Gordon L. Stuiber, and Zhangdui Zhong. "Non-stationary mobile-to-mobile channel modeling using the Gauss-Markov mobility model." In Wireless Communications and Signal Processing (WCSP), 2017 9th International Conference on, pp. 1-6. IEEE, 2017.

28. Broyles, Dan, Abdul Jabbar, and James PG Sterbenz. "Design and analysis of a 3-D gauss-markov mobility model for highly-dynamic airborne networks." In Proceedings of the International Telemetering Conference (ITC),(San Diego, CA). 2010.

29. Li, Xianfeng, Tao Zhang, and Jianfeng Li. "A Particle Swarm Mobility Model for Flying Ad Hoc Networks." In GLOBECOM 2017-2017 IEEE Global Communications Conference, pp. 1-6. IEEE, 
2017.

30. Li, Xianfeng, and Tao Zhang. "STGM: A Spatiotemporally Correlated Group Mobility Model for Flying Ad Hoc Networks." In International Conference on Communicatins and Networking in China, pp. 391-400. Springer, Cham, 2016.

31. Bouachir, Ouns, AlinoeAbrassart, Fabien Garcia, and Nicolas Larrieu. "A mobility model for UAV ad hoc network." In Unmanned Aircraft Systems (ICUAS), 2014 International Conference on, pp. 383-388. IEEE, 2014.

32. Radu, Dan, Adrian Cretu, Benoît Parrein, Jiazi Yi, Camelia Avram, and Adina Aştilean. "Flying Ad Hoc Network for Emergency Applications Connected to a Fog System." In International Conference on Emerging Internetworking, Data \& Web Technologies, pp. 675-686. Springer, Cham, 2018.

33. Rosati, Stefano, Karol Krużelecki, Gregoire Heitz, Dario Floreano, and BixioRimoldi. "Dynamic routing for flying ad hoc networks." IEEE Transactions on Vehicular Technology 65, no. 3 (2016): 1690-1700.

34. Le, Michael, Joon-Sang Park, and Mario Gerla. "UAV assisted disruption tolerant routing." In Military Communications Conference, 2006. MILCOM 2006. IEEE, pp. 1-5. IEEE, 2006.

35. Messous, Mohamed-Ayoub, Sidi-Mohammed Senouci, and HichemSedjelmaci. "Network connectivity and area coverage for UAV fleet mobility model with energy constraint." In Wireless Communications and Networking Conference (WCNC), 2016 IEEE, pp. 1-6. IEEE, 2016.

36. Zacarias, Iulisloi, Luciano P. Gaspary, Andersonn Kohl, Ricardo QA Fernandes, Jorgito M. Stocchero, and Edison P. de Freitas. "Combining Software-Defined and Delay-Tolerant Approaches in Last-Mile Tactical Edge Networking." IEEE Communications Magazine 55, no. 10 (2017): 22-29.

37. Sánchez-García, J., D. G. Reina, and S. L. Toral. "A distributed PSO-based exploration algorithm for a UAV network assisting a disaster scenario." Future Generation Computer Systems 90 (2019): 129-148.

38. Karmakar, Gour, JoarderKamruzzaman, and Nusrat Nowsheen. "An efficient data delivery mechanism for AUV-based Ad hoc UASNs." Future Generation Computer Systems 86 (2018): 1193-1208.

39. Kerrache, Chaker Abdelaziz, EzedinBarka, NasreddineLagraa, and AbderrahmaneLakas. "Reputation-aware energy-efficient solution for FANET monitoring." In Wireless and Mobile Networking Conference (WMNC), 2017 10th IFIP, pp. 1-6. IEEE, 2017. 
40. De Vit, Antônio Rodrigo D., César Marcon, Raul Ceretta Nunes, Thais Webber, Gustavo Sanchez, and Carlos Oberdan Rolim. "Energy saving on DTN using trajectory inference model." In Proceedings of the 33rd Annual ACM Symposium on Applied Computing, pp. 2132-2135. ACM, 2018.

41. Zhou, Huan, Victor CM Leung, Chunsheng Zhu, Shouzhi Xu, and Jialu Fan. "Predicting temporal social contact patterns for data forwarding in opportunistic mobile networks." IEEE Transactions on Vehicular Technology 66, no. 11 (2017): 10372-10383.

42. Guo, Shuo, Liang He, Yu Gu, Bo Jiang, and Tian He. "Opportunistic flooding in low-duty-cycle wireless sensor networks with unreliable links." IEEE Transactions on Computers 63, no. 11 (2014): 2787-2802.

43. Zhang, Xiaolan, Giovanni Neglia, Jim Kurose, and Don Towsley. "Performance modeling of epidemic routing." Computer Networks 51, no. 10 (2007): 2867-2891.

44. Lindgren, Anders, AvriDoria, Elwyn Davies, and SamoGrasic. Probabilistic routing protocol for intermittently connected networks. No. RFC 6693. 2012.

45. Spyropoulos, Thrasyvoulos, Konstantinos Psounis, and Cauligi S. Raghavendra. "Spray and wait: an efficient routing scheme for intermittently connected mobile networks." In Proceedings of the 2005 ACM SIGCOMM workshop on Delay-tolerant networking, pp. 252-259. ACM, 2005.

46. Camp, Tracy, Jeff Boleng, and Vanessa Davies. "A survey of mobility models for ad hoc network research." Wireless communications and mobile computing 2, no. 5 (2002): 483-502.

47. González, Ramón, Paramsothy Jayakumar, and Karl Iagnemma. "Stochastic mobility prediction of ground vehicles over large spatial regions: a geostatistical approach." Autonomous Robots 41, no. 2 (2017): 311-331.

48. Keränen, Ari, Jörg Ott, and TeemuKärkkäinen. "The ONE simulator for DTN protocol evaluation." In Proceedings of the 2nd international conference on simulation tools and techniques, p. 55. ICST (Institute for Computer Sciences, Social-Informatics and Telecommunications Engineering), 2009.

49. Burgess, John, Brian Gallagher, David Jensen, and Brian Neil Levine. "Maxprop: Routing for vehicle-based disruption-tolerant networks." In INFOCOM 2006. 25th IEEE International Conference on Computer Communications. Proceedings, pp. 1-11. IEEE, 2006.

50. Ahmad, Khaleel, Muneera Fathima, Vishal Jain, and Afrah Fathima. "FUZZY-Prophet: a novel routing protocol for opportunistic network." International Journal of Information Technology 9, no. 2 (2017): 121-127.

51. Abdelkader, Tamer, Kshirasagar Naik, Amiya Nayak, Nishith Goel, and Vineet Srivastava. "A performance comparison of delay-tolerant network routing protocols." IEEE Network 30, no. 2 (2016): 46-53. 
52. Cabacas, Regina A., Hideaki Nakamura, and In-Ho Ra. "Energy consumption analysis of delay tolerant network routing protocols." International Journal of Software Engineering and Its Applications 8, no. 2 (2014): 1-10.

53. Ferreira, Danielle L., Bruno Astuto A. Nunes, and Katia Obraczka. "Scale-Free Properties of Human Mobility and Applications to Intelligent Transportation Systems." IEEE Transactions on Intelligent Transportation Systems 19, no. 11 (2018): 3736-3748.

54. Basta, Nardine, Amal ElNahas, Hans-Peter Grossmann, and Slim Abdennadher. "Guess where I go?: A Mobility Predictor for Smart Vehicles." In Proceedings of the 17th International Conference on Mobile and Ubiquitous Multimedia, pp. 93-102. ACM, 2018.

55. Ghouti, Lahouari. "Mobility prediction in mobile ad hoc networks using neural learning machines." Simulation Modelling Practice and Theory 66 (2016): 104-121. 


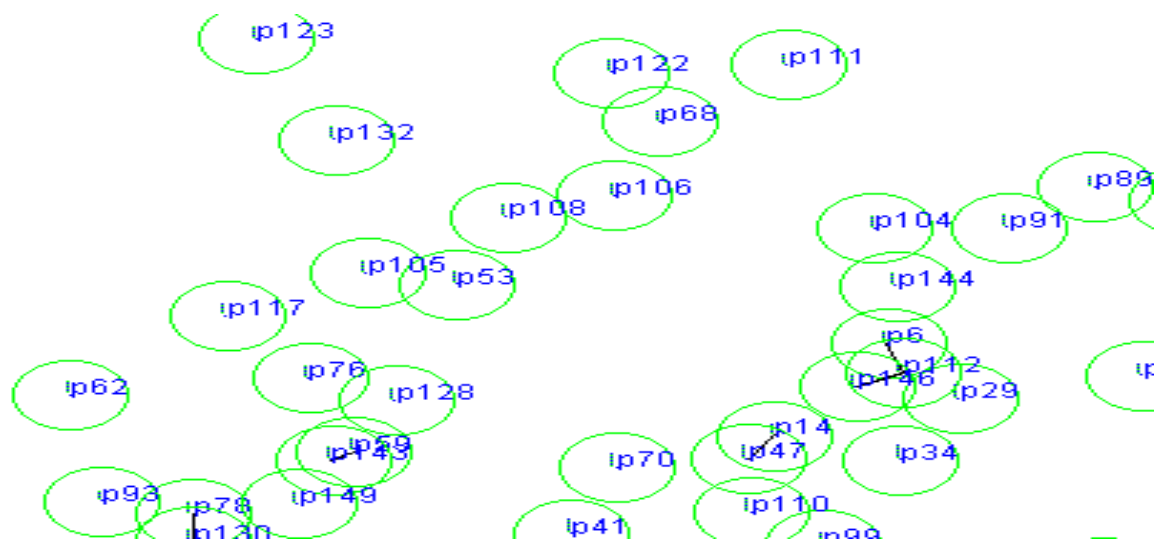

Fig 1. Snapshot of the used simulation environment.

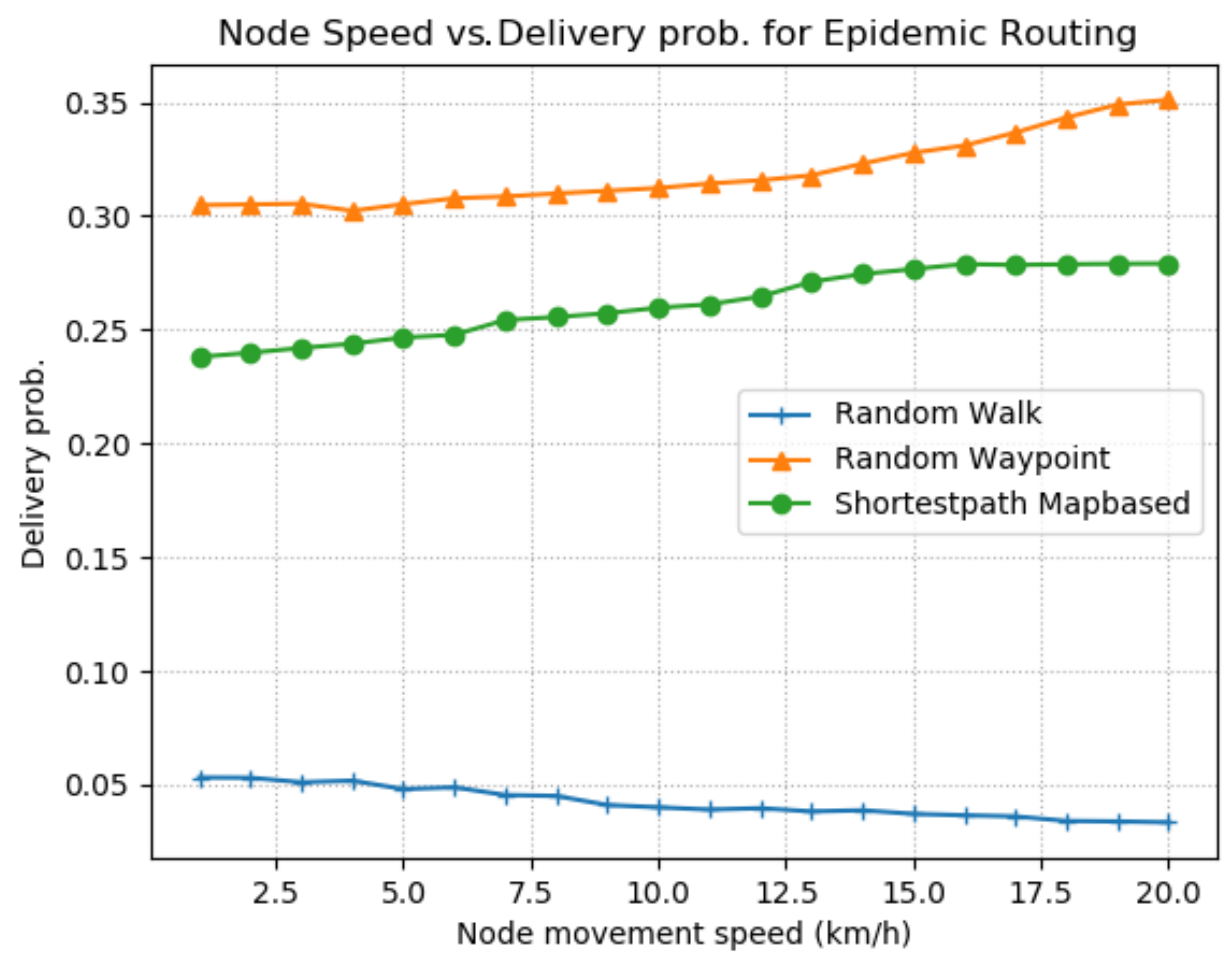

Fig 2. Node speed vs. Delivery Probability: epidemic routing for proposed Random Walk, Random Waypoint, and Shortest path Map based movement models. 


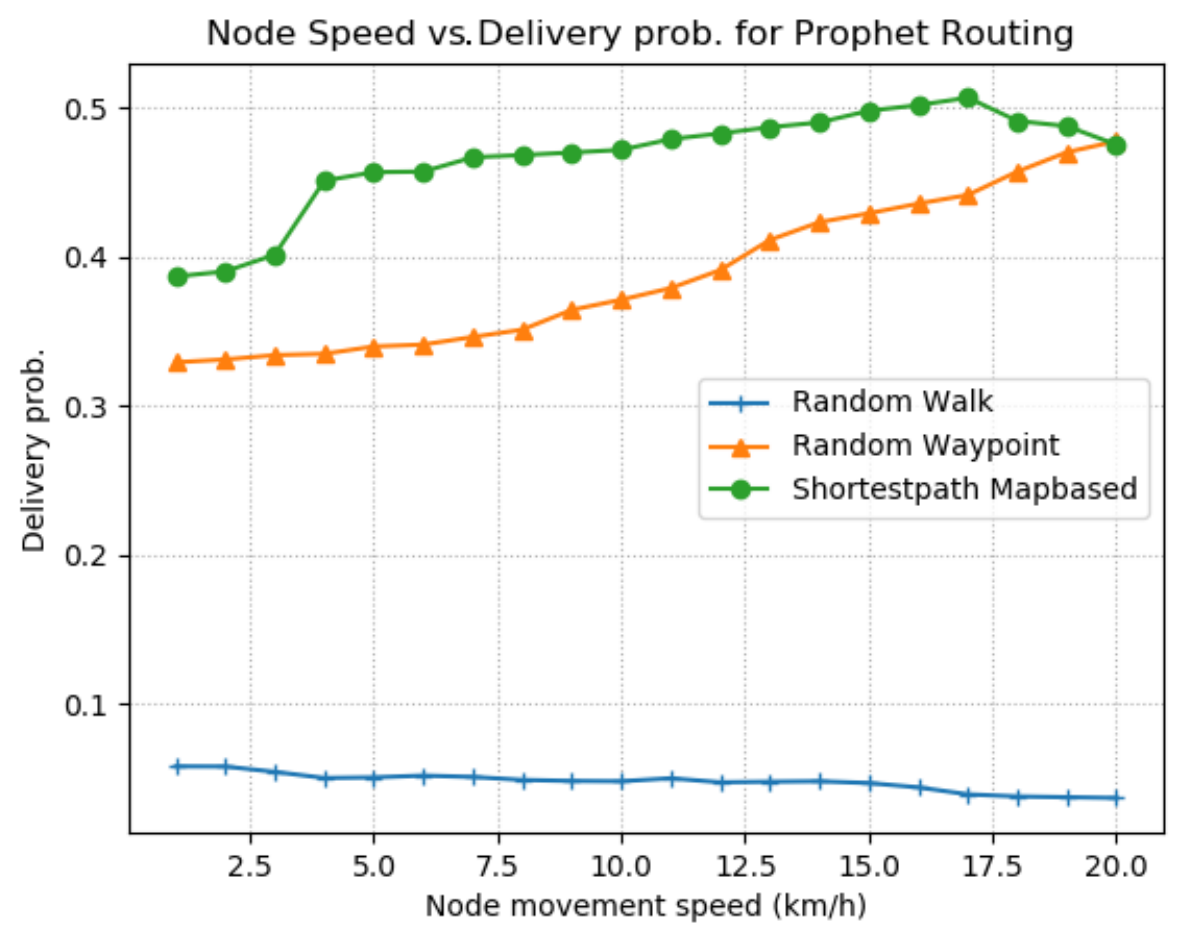

Fig 3.Node speed vs. Delivery Probability: Prophet routing for proposed Random Walk, Random Waypoint, and Shortest path Map based movement models.

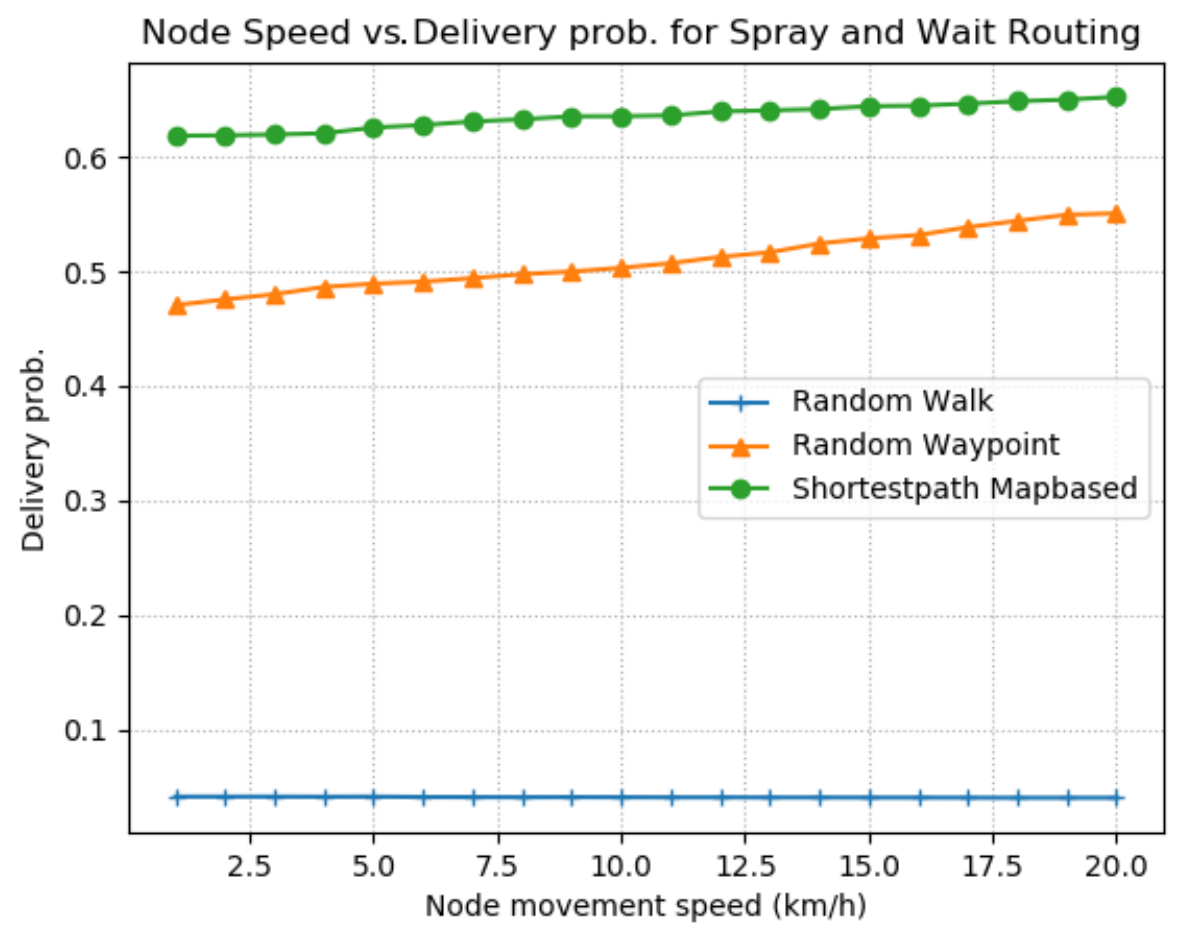

Fig 4. Node speed vs. Delivery Probability: Spray and Wait routing for proposed Random Walk, Random Waypoint, and Shortest path Map based movement models. 


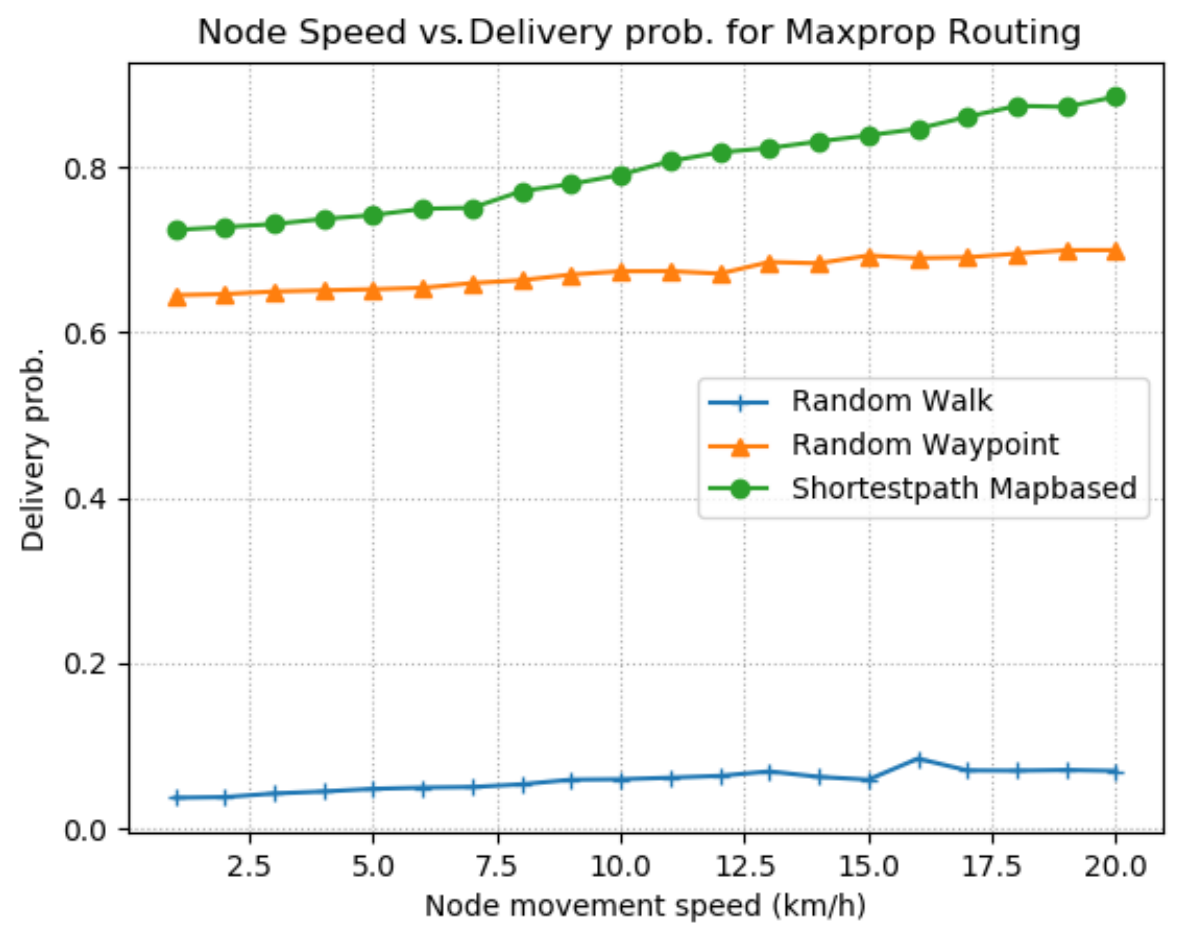

Fig 5. Node speed vs. Delivery Probability: MaxProp routing for proposed Random Walk, Random Waypoint, and Shortest path Map based movement models.

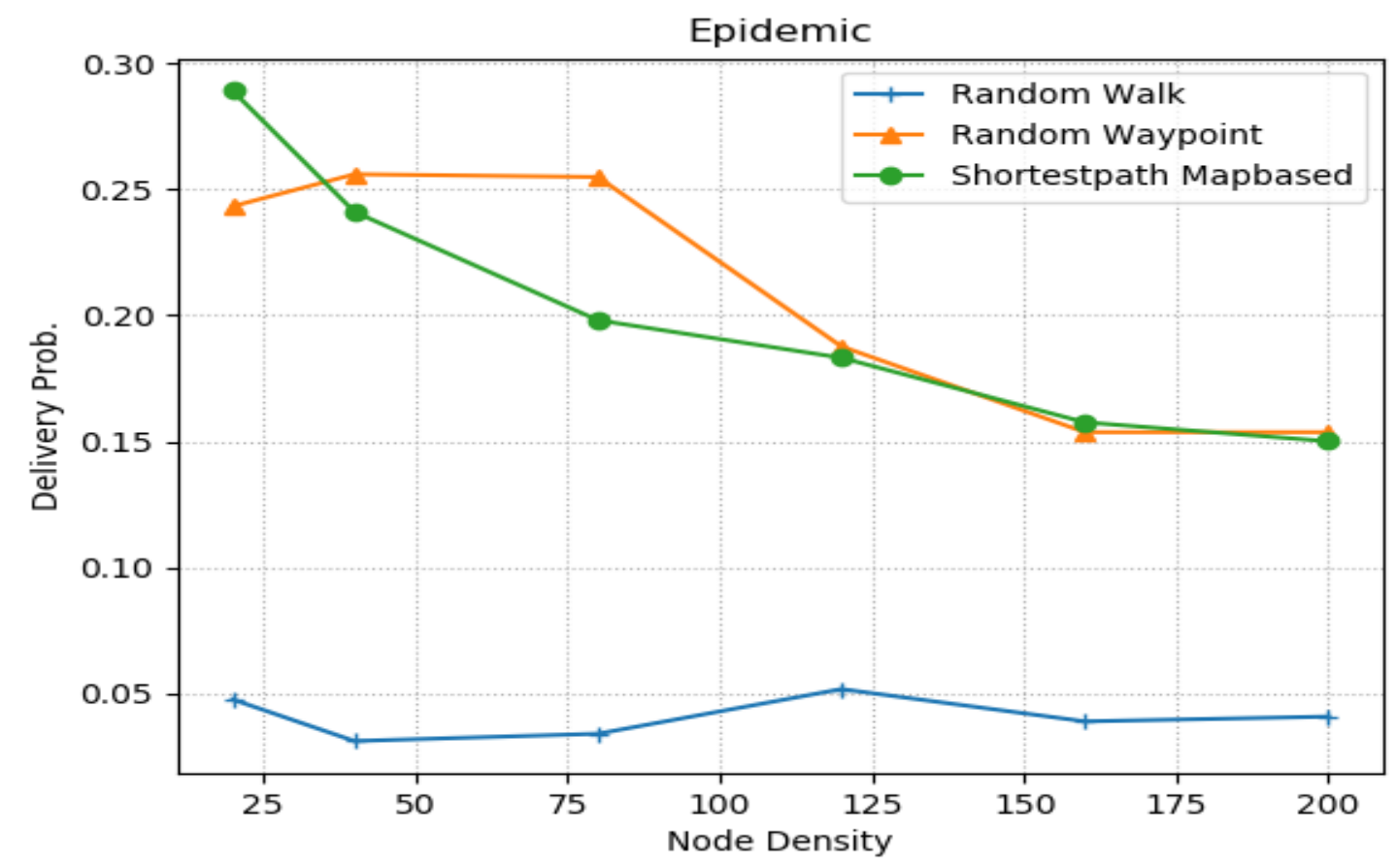

Fig 6. Node density vs. delivery probability: Epidemic routing for proposed Random Walk, Random Waypoint, and Shortest path Map based movement models. 


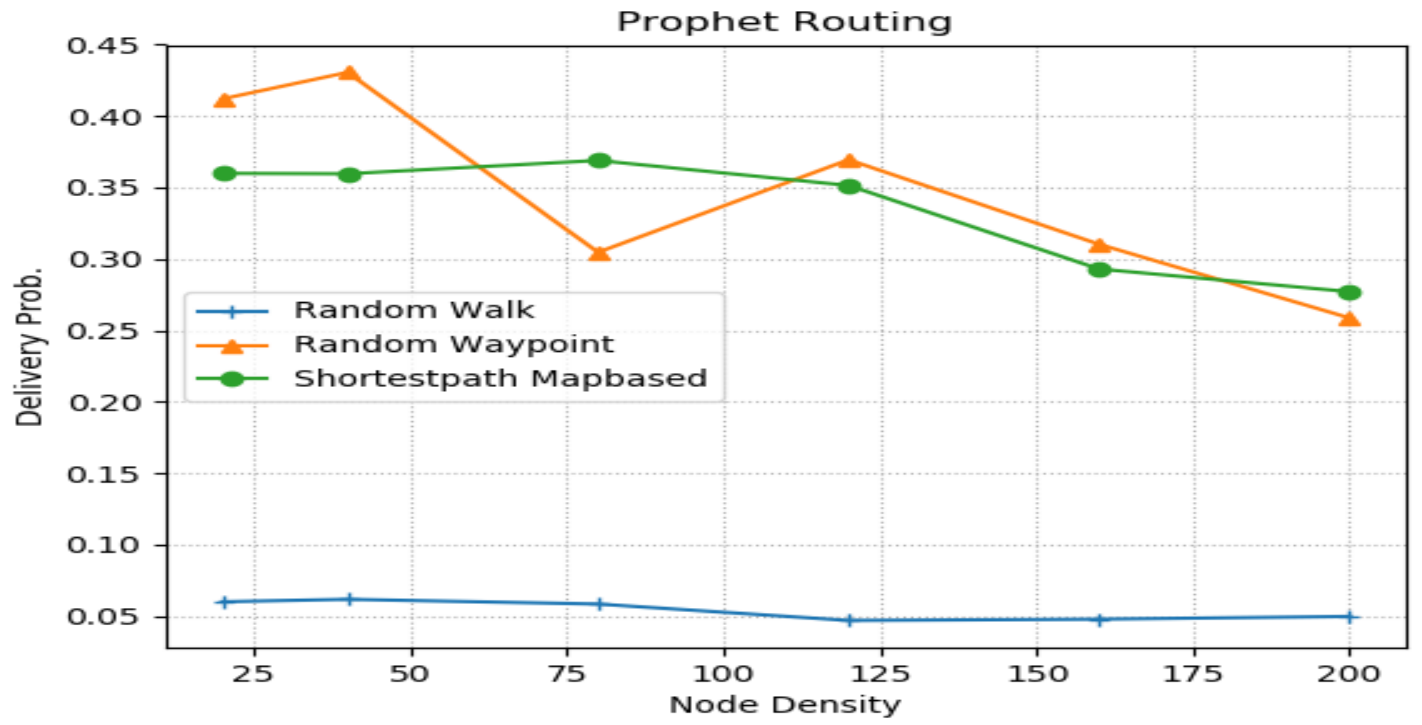

Fig 7. Node density vs. delivery probability: Prophet routing for proposed Random Walk, Random Waypoint, and Shortest path Map based movement models.

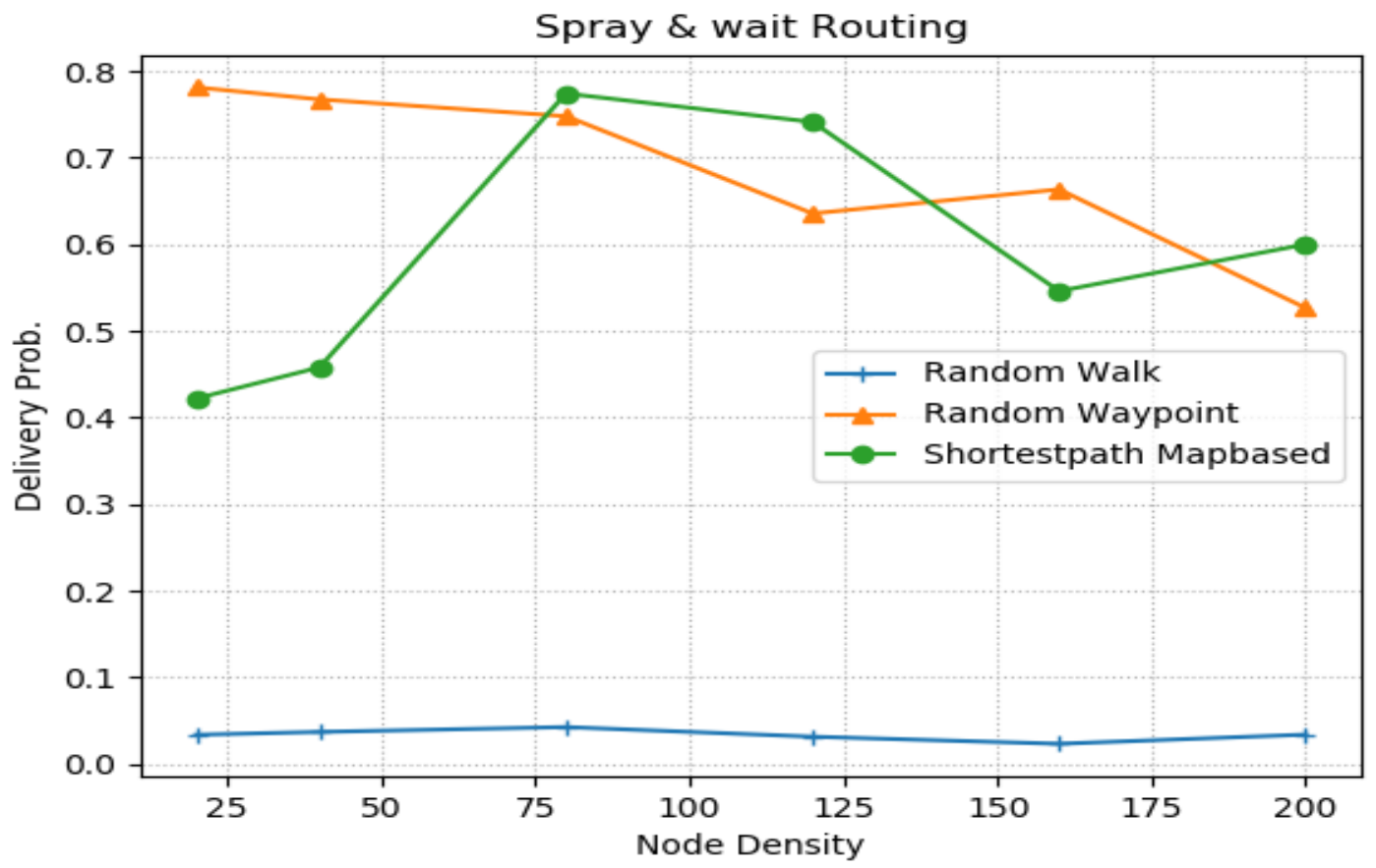

Fig 8. Node density vs. delivery probability: Spray and wait routing for proposed Random Walk, Random Waypoint, and Shortest path Map based movement models. 


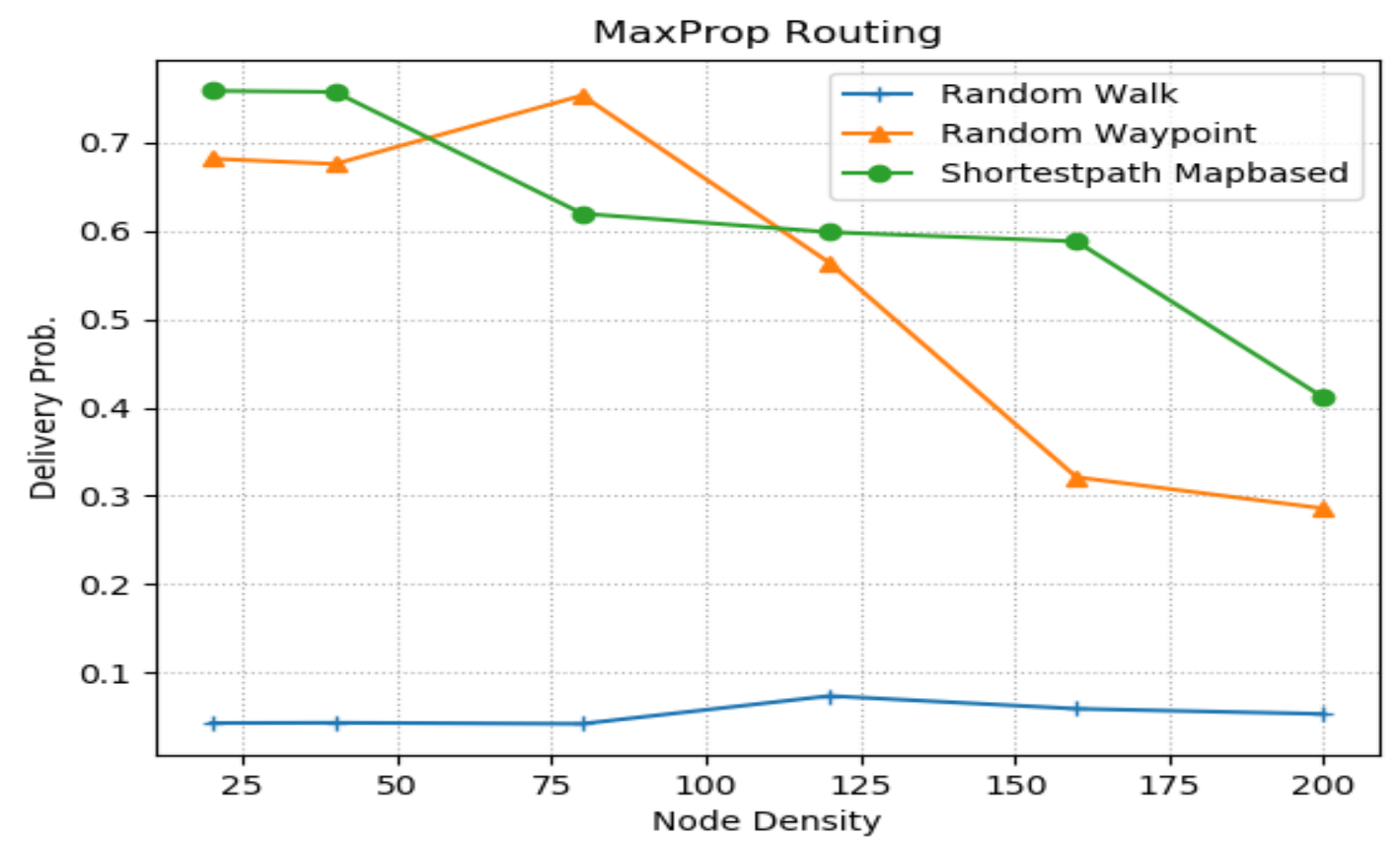

Fig 9. Node density vs. delivery probability: MaxProp routing for proposed Random Walk, Random Waypoint, and Shortest path Map based movement models.

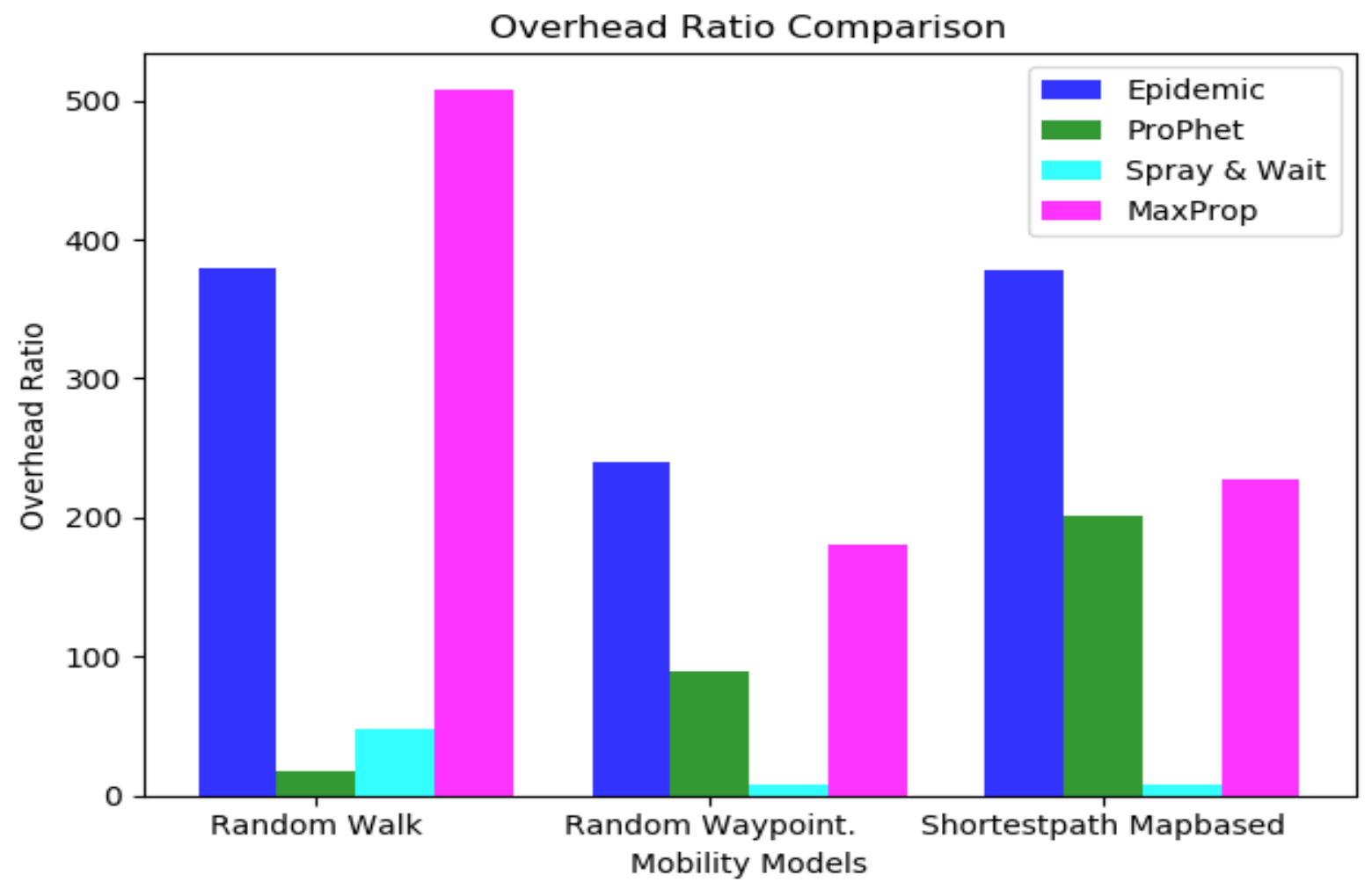

Fig 10. Comparison of message overhead ratio for the four different routing strategies underproposed mobility models. 


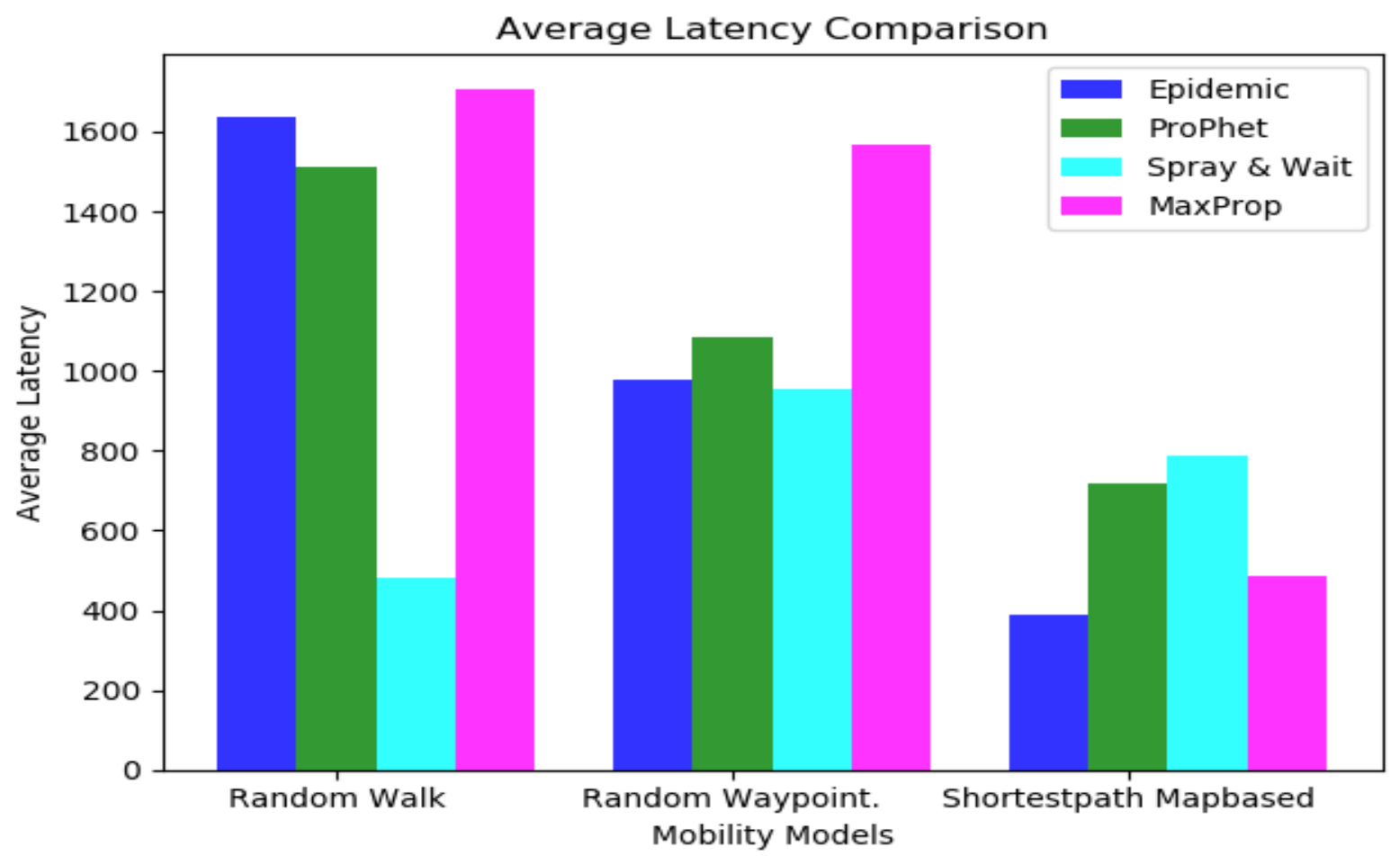

Fig 11. Comparison of Average message delivery latency for the four different routing strategy under proposed mobility models.

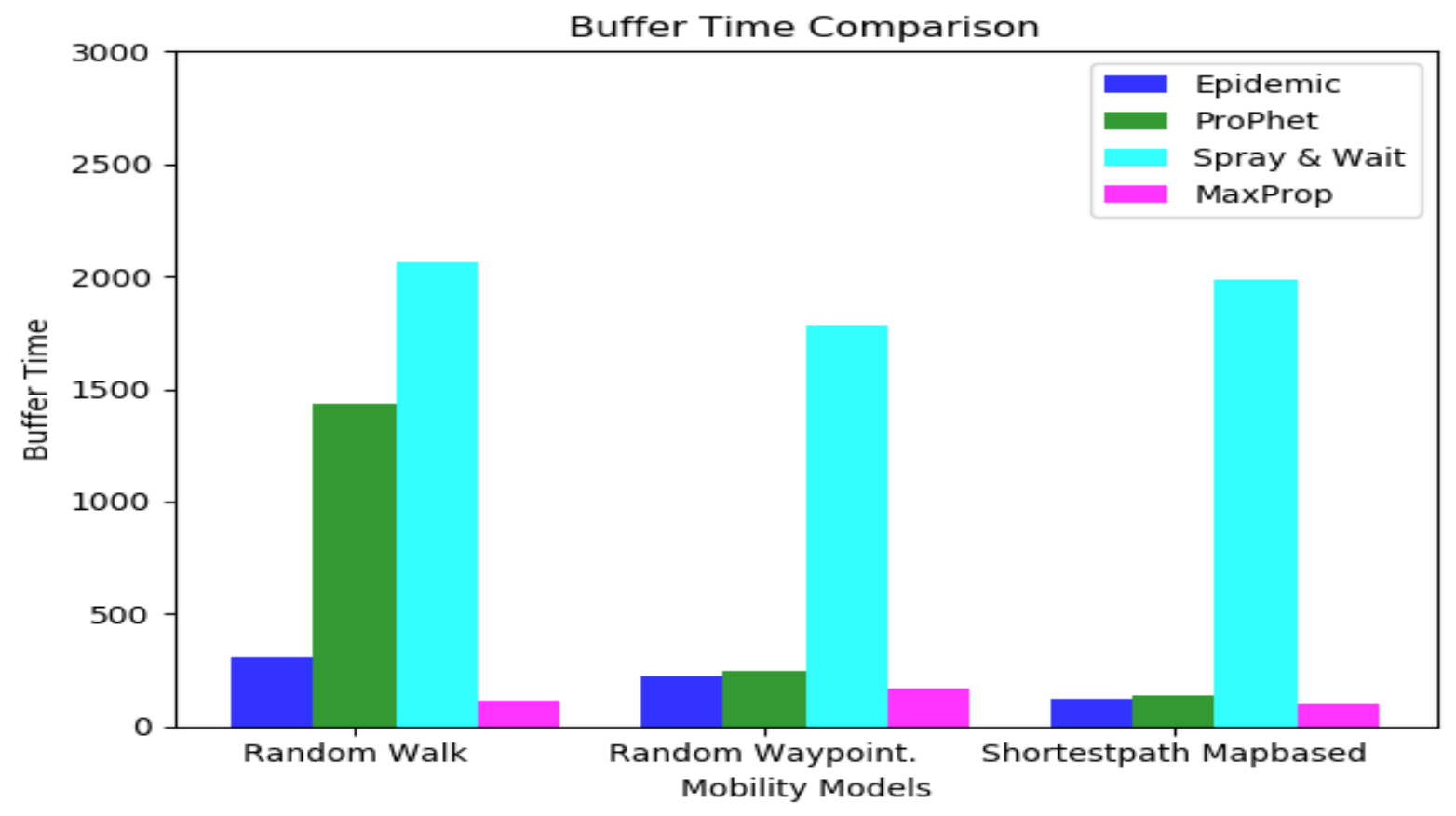

Fig 12. Comparison of Average Buffer time for different routing strategies under proposed mobility models. 


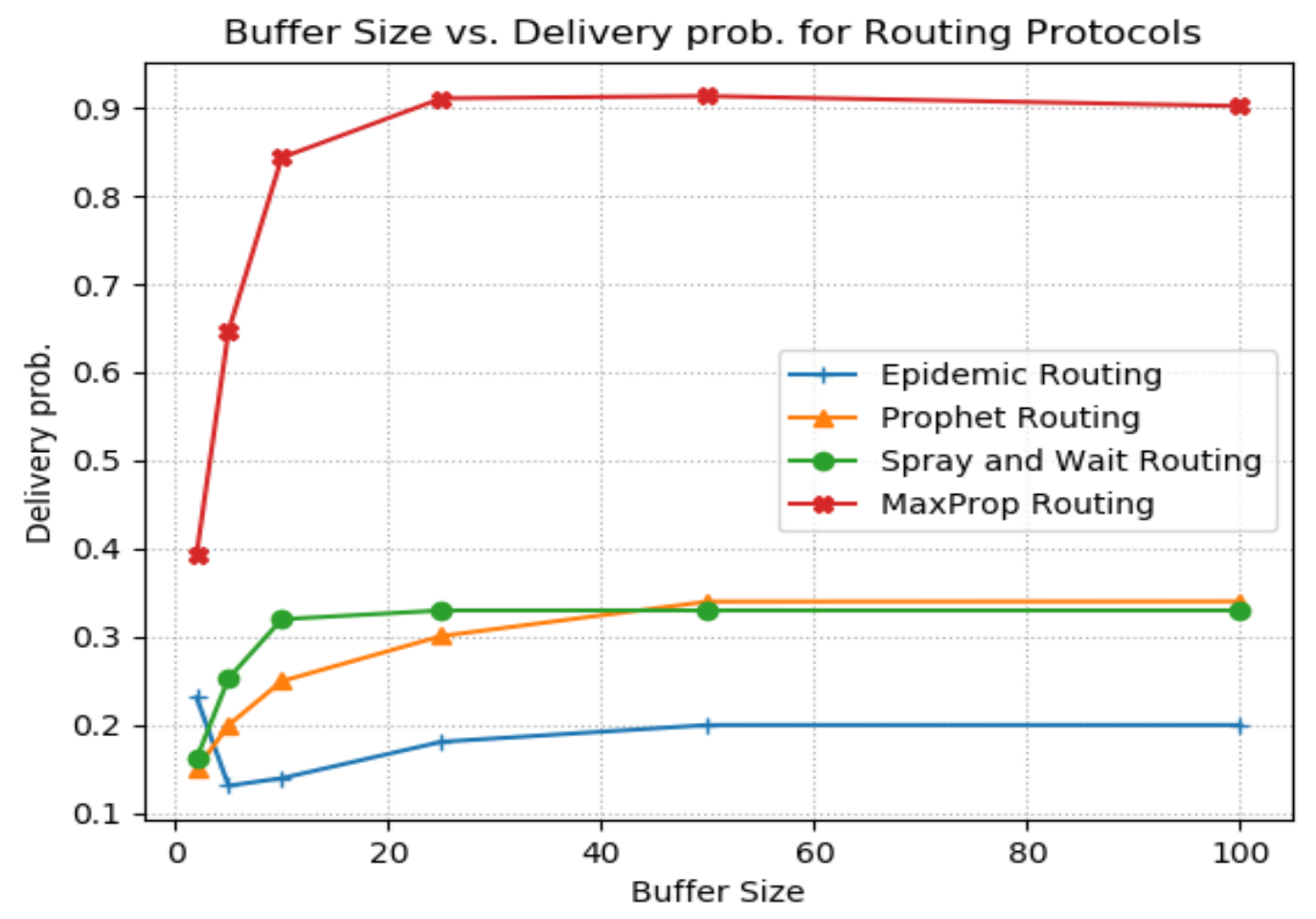

(a)

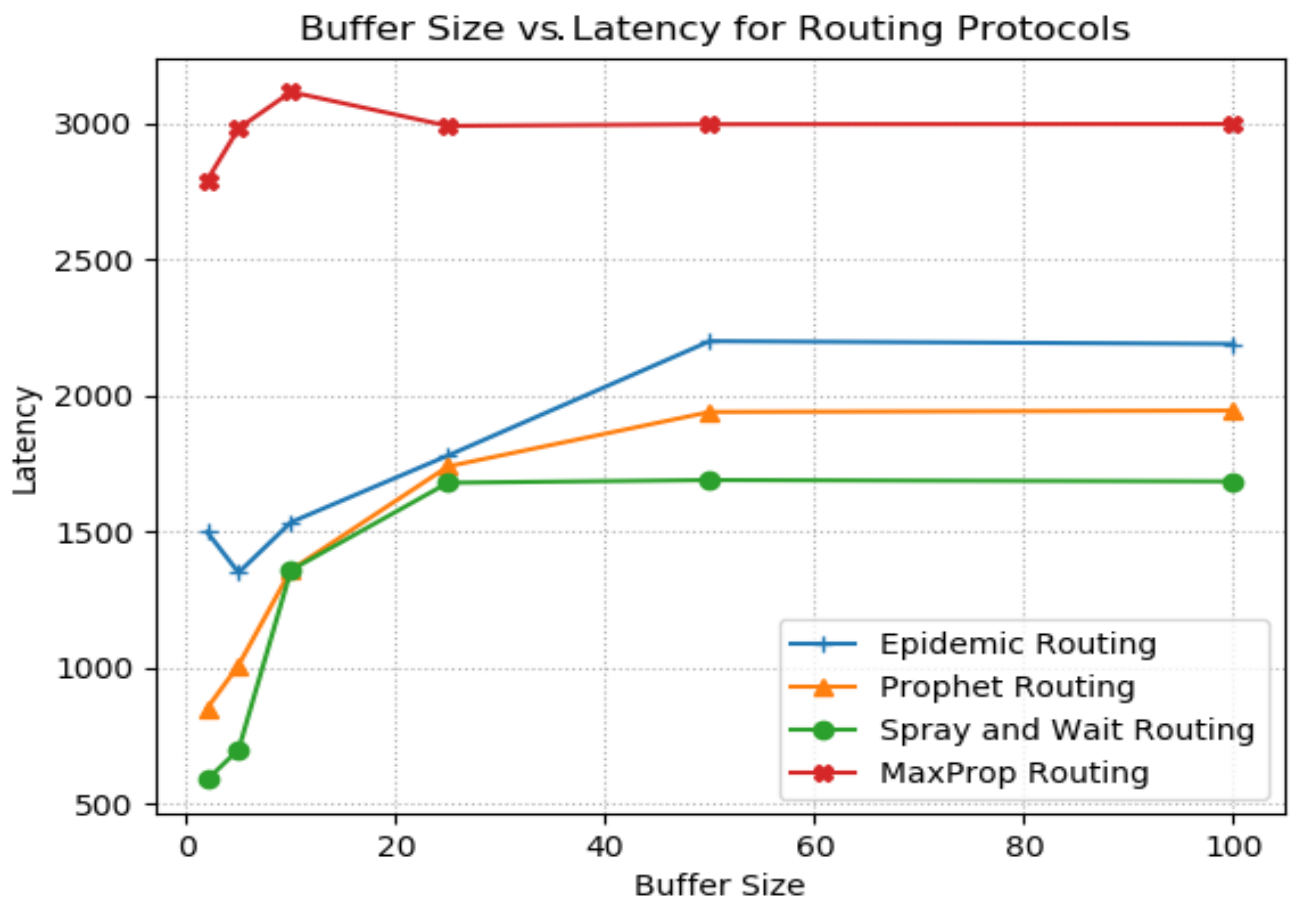

(b)

Fig 13. (a) Delivery Probability vs. Buffer size and (b) Latency vs. Buffer Size for the four Routing strategies under study. 
Avarage delivery prob. for all routing achived by mobility models.

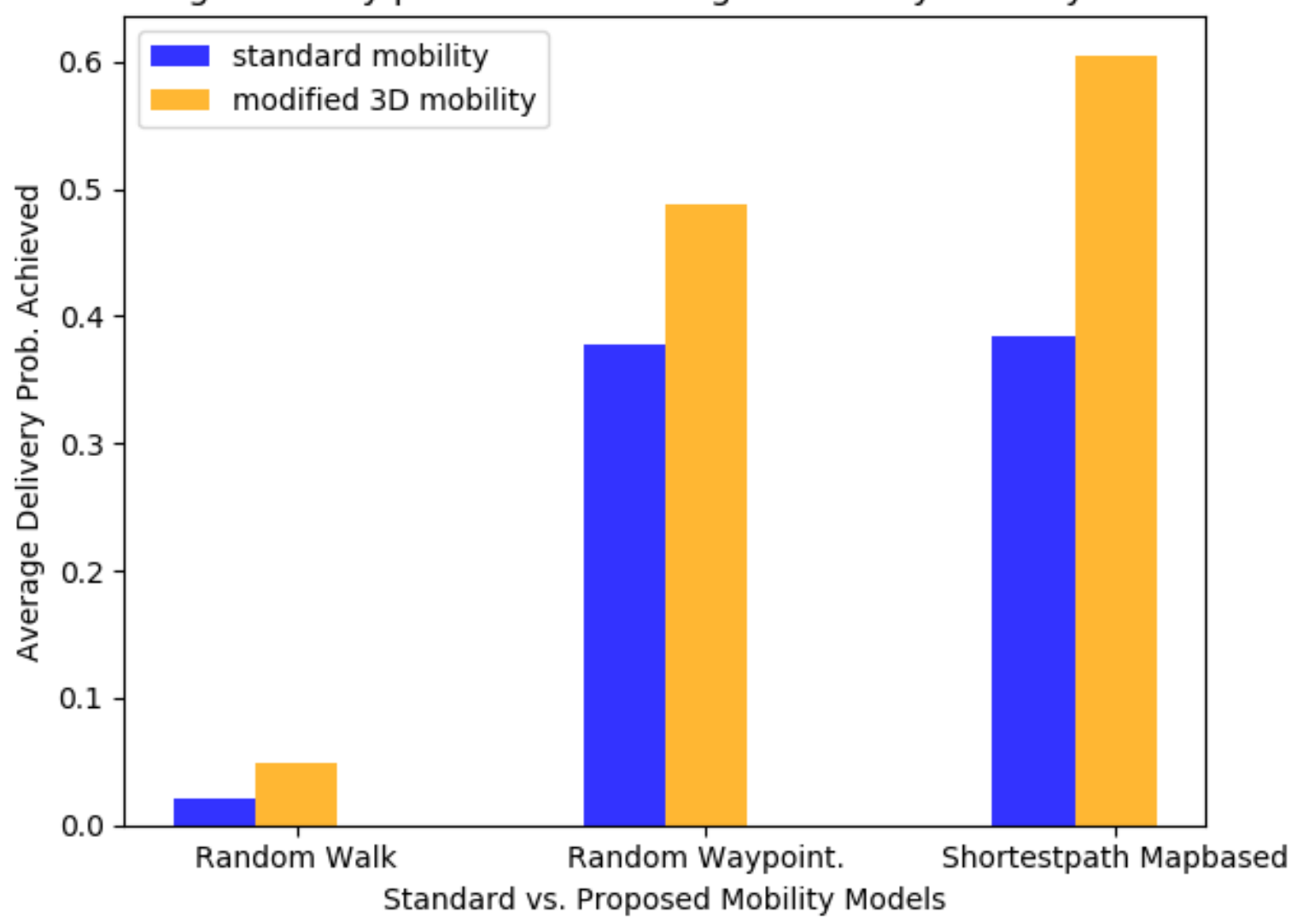

Fin 14. Message deliverv prob. Comparison for standard and modified mobilitv 


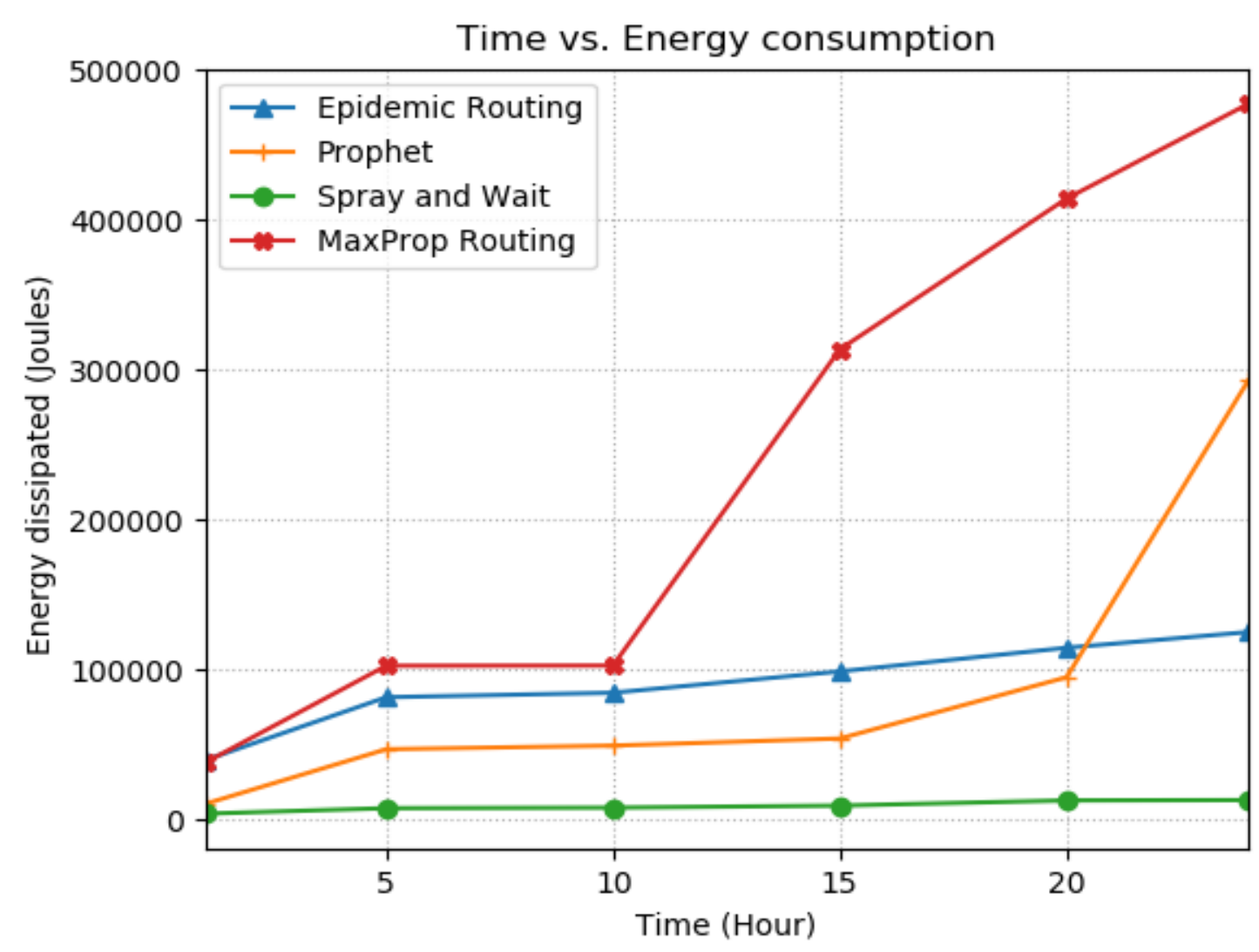

Figure 15. Energy consumption w.r.t time for routing algorithms. 


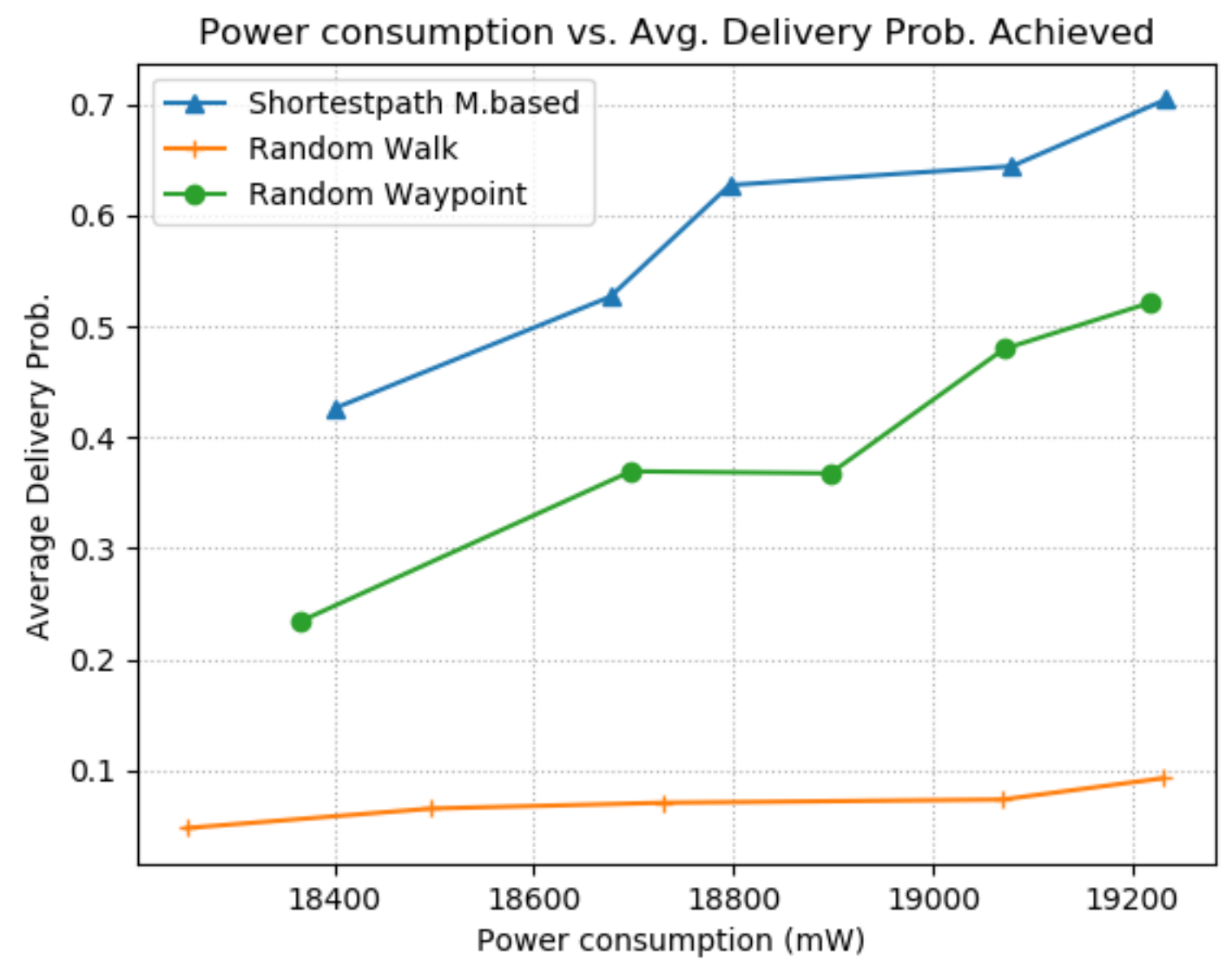

Fig 16. Power Dissipation vs. delivery probability achieved by the proposed mobility model. 
Table 1. Used standard simulation parameters

\begin{tabular}{|c|c|}
\hline Parameter & Value \\
\hline Simulation Time & 43200 (12 hours) \\
\hline Number of FANET nodes & 40 \\
\hline Transmission range for \\
IEEE802.11b/g/n interface
\end{tabular}

Table 2. Used simulation specific parameters for routing protocol

\begin{tabular}{|c|c|}
\hline Parameters & Value \\
\hline Prophet (Seconds in time unit) & $30 \mathrm{sec}$ \\
\hline Spray and wait (number of copies) & 3 \\
\hline Spray and wait (Binary mode) & True \\
\hline Epidemic & N/A \\
\hline MaxProp & N/A \\
\hline
\end{tabular}

Table 3: Used Simulation Parameter Settings for Energy

\begin{tabular}{|l|l|}
\hline \multicolumn{1}{|c|}{ Variable } & \multicolumn{1}{c|}{ Value } \\
\hline Total Power & $520000 \mathrm{mAh}$ \\
\hline Initial Energy & $6.17 \times 10^{6} \mathrm{~J}$ \\
\hline Scan Energy & $0.014 \mathrm{~J} / \mathrm{s}$ \\
\hline Scan Interval & $0.5 \mathrm{sec}$ \\
\hline Transmission Energy & $0.13365 \mathrm{~J} / \mathrm{s}$ \\
\hline
\end{tabular}


Amartya Mukherjee is an assistant professor at the Institute of Engineering and Management, Salt Lake, Kolkata, India. He holds a bachelor's degree in computer science and engineering from West Bengal University of Technology and a master's degree in computer science and engineering from the National Institute of Technology, Durgapur, West Bengal, India. His primary research interest is in embedded application development, including mobile ad hoc networking, aerial robotics, and Internet of Things and machine learning. He has written several research articles in the field of wireless networking and embedded systems. His book Embedded systems and robotics with open source tools is one of the bestselling books in the field of embedded application development.

Nilanjan Day was born in Kolkata, India, in 1984. He received his B.Tech. degree in Information Technology from West Bengal University of Technology in 2005, M.Tech. in Information Technology in 2011 from the same University and Ph.D. in digital image processing in 2015 from Jadavpur University, India. In 2011, he was appointed as an Asst. Professor in the Department of Information Technology at JIS College of Engineering, Kalyani, India followed by Bengal College of Engineering College, Durgapur, India in 2014. He is now employed as an Asst. Professor in Department of Information Technology, Techno India College of Technology, India. His research topic is signal processing, machine learning and information security. He is an Associate Editor of IEEE ACCESS and is currently the Editor in-Chief of the International Journal of Ambient Computing and Intelligence, International Journal of Rough Sets and Data Analysis, International Journal of Natural Computing Research, Co-Editorin-Chief of International Journal of Synthetic Emotion and Series Editor of Advances in Geospatial Technologies Book Series.

Rajesh Kumar received a Bachelor of Technology Degree with Honours from National Institute of Technology, Kurukshetra, India, in 1994. He also earned a Masters of Engineering Degree with Honours from the Malaviya National Institute of Technology, Jaipur, India in 1997; he earned a PhD. Degree from the Malaviya National Institute of Technology, Jaipur and University of Rajasthan, Jaipur in 2005. He was awarded Post Doctorate Research Fellow in the Department of Electrical and Computer Engineering at the National University of Singapore (NUS), Singapore, from 2009 to 2011. He joined the Department of Electrical Engineering at the Malaviya National Institute of Technology, Jaipur, India as a Lecturer in 1995. He is currently serving as Professor and Head. He is also adjunct faculty to Centre of Energy and Environment at Malaviya National Institute of Technology, Jaipur, India. Dr. Kumar has carried out extensive research in various areas of theory and practice of intelligent systems, bio and nature inspired algorithms, smart grid, power electronics, power management, applications of AI to image processing and robotics. He has published more than 450 papers in international refereed journals and conferences. He has received and published 12 patents. He has supervised $15 \mathrm{PhD}$ and 35 Master thesis. He has delivered more than 100 expert talks in various conferences and 
workshops. Dr. Kumar has won the Career Award for Young Teachers, Government of India in 2000. He received 06 best thesis awards, 05 academic awards, 12 best paper awards, 04 professional awards and 30 student awards. He is Vice Chairman IEEE Rajasthan Sub Section and Executive Member IEEE PES-IAS Delhi Chapter and Computer Society of India, Rajasthan Section. He is Associate Editor of IEEE ITeN (Industrial Electronics Technology News), Associate Editor, Swarm and Evolutionary Computation, Associate Editor, IET Renewable and Power Generation, Associate Editor, IET Power Electronics, Deputy Editor-in-Chief, CAAI Transactions on Intelligence Technology, Associate Editor, International Journal of Bio Inspired Computing. He is Editorial Member of more than 15 Journals. Dr. Kumar is also Senior Member IEEE (USA), Fellow IET (UK), Fellow IE (INDIA), Fellow IETE, Life Member CSI, Senior Member IEANG and Life Member ISTE.

Bijaya Ketan Panigrahi received the Ph.D. degree from Sambalpur University, Sambalpur, India, in 2004. He is a Professor with the Department of Electrical Engineering, Indian Institute of Technology (IIT), New Delhi, India.

His research interests include digital signal processing, power quality assessment, and application of soft computing techniques to power system. He serves as Associate Editor of IEEE SYSTEMS.

\begin{abstract}
Aboul Ella Hassanien received his B.Sc. with honours in 1986 and M.Sc degree in 1993, both from Ain Shams University, Faculty of Science, Pure Mathematics and Computer Science Department, Cairo, Egypt. On September 1998, he received his doctoral degree from the Department of Computer Science, Graduate School of Science \& Engineering, Tokyo Institute of Technology, Japan. He is a Full Professor at Cairo University, Faculty of Computer and Information, IT Department. Professor Aboul Ella is the founder and chair the scientific research group in Egypt (www.egyptscience.net/).

He has authored/coauthored over 380 research publications in peer-reviewed reputed journals, book chapters and conference proceedings. He has served as the general chair, co-chair, program chair, program committee member of various international conferences and reviewer for various international journals. His research interests include, Computational intelligence, medical image analysis, security, animal identification and multimedia data mining.
\end{abstract}

João Manuel R. S. Tavares graduated in Mechanical Engineering atthe Universidade do Porto, Portugal in 1992. He also earnedhis M.Sc. degree and Ph.D. degree in Electrical and Computer Engineering from the Universidade do Porto in 1995 and 2001, and attained hisHabilitation in Mechanical Engineering in 2015. Heisa seniorresearcherattheInstituto de Ciência e Inovação em 
Engenharia Mecânica e Engenharia Industrial (INEGI) andAssociate Professor attheDepartmentofMechanicalEngineering(DEMec) oftheFaculdade de Engenharia daUniversidade do Porto (FEUP).

João Tavares is co-editor of more than 40 books, co-author of more than 35 book chapters, 600 articles in international and national journals and conferences, and 3 international and 2 national patents. He has been a committee member of several international and national journals and conferences, is co-founder and co-editor of the book series "Lecture Notes in Computational Vision and Biomechanics" published by Springer, founder and Editor-in-Chief of the journal "Computer Methods in Biomechanics and Biomedical Engineering: Imaging \& Visualization" published by Taylor \& Francis, and co-founder and co-chair of the international conference series: CompIMAGE, ECCOMASVipIMAGE, ICCEBS and BioDental. Additionally, he has been (co-)supervisor of several MSc and $\mathrm{PhD}$ thesis and supervisor of several post-doc projects, and has participated inmanyscientific projects both as researcher and as scientific coordinator. His main research areas include computational vision, medical imaging, computational mechanics, scientific visualization, human-computer interaction and new product development. (More information can be found at: www.fe.up.pt/ tavares) 


\section{Authors Photo}

Amartya Mukherjee

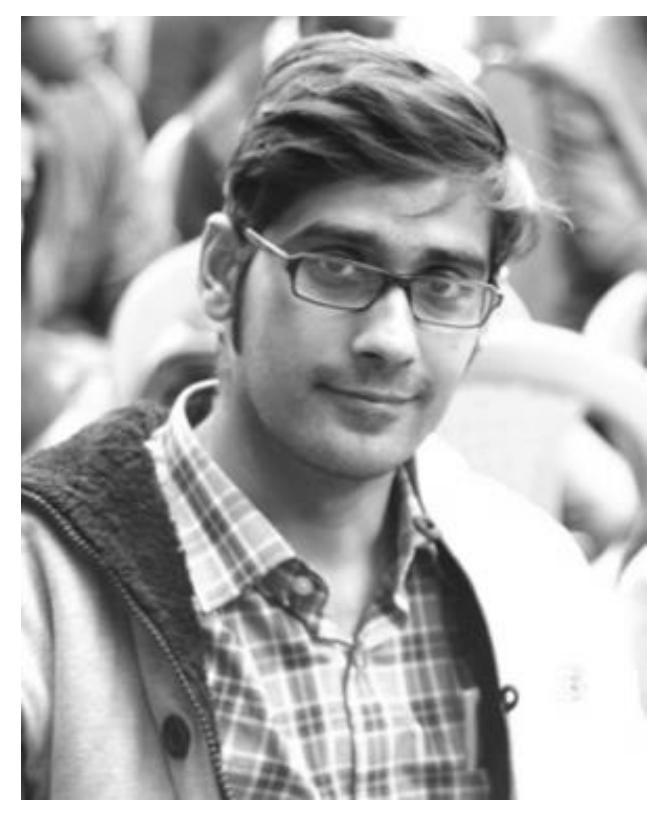

Nilanjan Dey

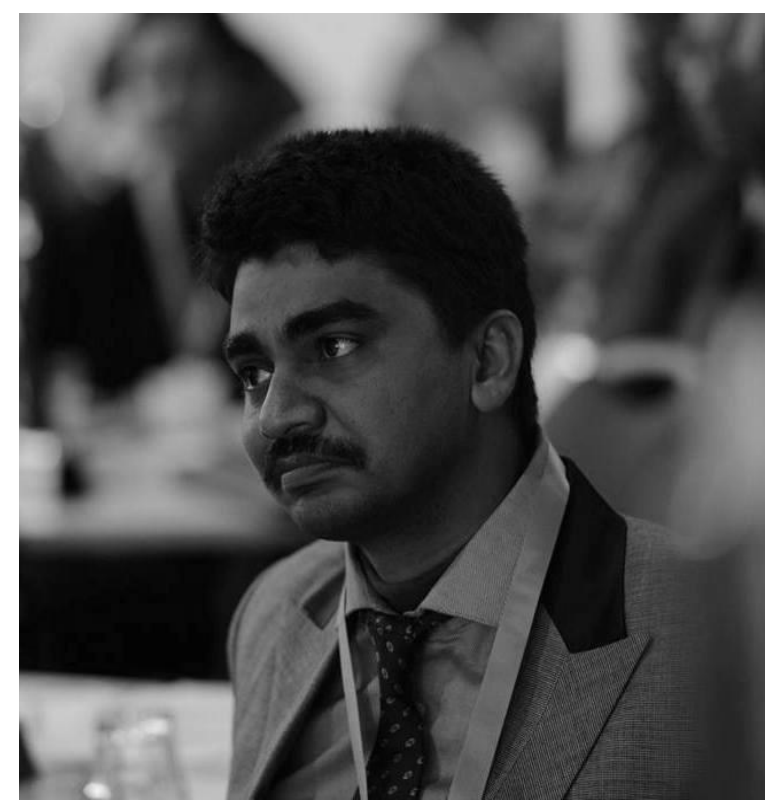




$$
\text { P }
$$


Aboul Ella Hassanien

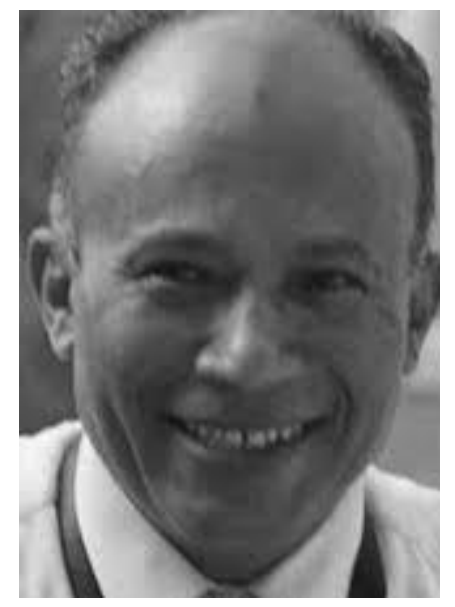

João Manuel R.S. Tavares

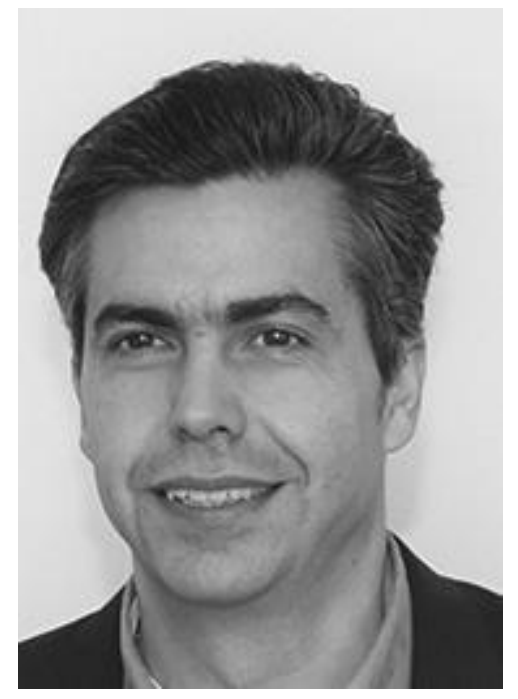

